\title{
Large-scale molecular shocks in galaxies: the SiO interferometer map of IC $342^{\star}$
}

\author{
A. Usero ${ }^{1}$, S. García-Burillo ${ }^{1}$, J. Martín-Pintado ${ }^{2}$, A. Fuente ${ }^{1}$, and R. Neri ${ }^{3}$ \\ 1 Observatorio Astronómico Nacional (OAN) - Observatorio de Madrid, C/ Alfonso XII 3, 28014 Madrid, Spain \\ e-mail: [a.usero;s.gburillo;a.fuente]@oan.es \\ 2 Instituto de Estructura de la Materia, DAMIR-CSIC, C/ Serrano 121, 28006 Madrid, Spain \\ e-mail: jmartin.pintado@iem.cfmac.csic.es \\ 3 IRAM, 300 rue de la Piscine, Domaine Universitaire, 38406 St. Martin d'Hères Cedex, France \\ e-mail: neri@iram.fr
}

Received 11 August 2005 / Accepted 6 October 2005

\begin{abstract}
We present the first high-resolution $\left(55^{\prime \prime} 6 \times 55^{\prime \prime} 1\right)$ images of the emission of silicon monoxide $(\mathrm{SiO})$ in the nucleus of the nearby spiral IC 342 , obtained with the IRAM Plateau de Bure Interferometer (PdBI). Using a two-field mosaic, we have simultaneously mapped the emission of the $\mathrm{SiO}(v=0, J=2-1)$ and $\mathrm{H}^{13} \mathrm{CO}^{+}(J=1-0)$ lines in a region of $\sim 0.9 \mathrm{kpc} \times 1.3 \mathrm{kpc}(\mathrm{RA} \times$ Dec $)$ centered around the nucleus of IC 342 . The bulk of the emission in the two lines comes from a $\sim 290 \mathrm{pc}$ spiral arm located to the North and a central component that forms the southern ridge of a $r \sim 80 \mathrm{pc}$ nuclear ring that was identified in other interferometer maps of the galaxy. We detect continuum emission at $86.8 \mathrm{GHz}$ in a $\sim 80-180 \mathrm{pc}$ central source. The continuum emission, dominated by thermal free-free bremsstrahlung, is mostly anticorrelated with the observed distribution of $\mathrm{SiO}$ clouds. The $\mathrm{SiO}-\mathrm{to}-\mathrm{H}^{13} \mathrm{CO}^{+}$intensity ratio is seen to increase by an order of magnitude from the nuclear ring ( 0.3) to the spiral arm ( 3.3). Furthermore the gas kinematics show significant differences between $\mathrm{SiO}$ and $\mathrm{H}^{13} \mathrm{CO}^{+}$over the spiral arm, where the linewidths of $\mathrm{SiO}$ are a factor of 2 larger than those of $\mathrm{H}^{13} \mathrm{CO}^{+}$. The average abundance of SiO in the inner $r \sim 320 \mathrm{pc}$ of IC 342 is $X(\mathrm{SiO}) \gtrsim 2 \times 10^{-10}$. This shows that shock chemistry is at work in the inner molecular gas reservoir of IC 342 .

To shed light on the nature of shocks in IC 342, we have compared the emission of SiO with another tracer of molecular shocks: the emission of methanol $\left(\mathrm{CH}_{3} \mathrm{OH}\right)$. We find that the significant difference of the abundance of $\mathrm{SiO}$ measured between the spiral $\operatorname{arm}\left(X(\mathrm{SiO}) \sim \mathrm{a}\right.$ few $\left.10^{-9}\right)$ and the nuclear ring $\left(X(\mathrm{SiO}) \sim 10^{-10}\right)$ is not echoed by a comparable variation in the $\mathrm{SiO}-$ to- $\mathrm{CH}_{3} \mathrm{OH}$ intensity ratio. This implies that the typical shock velocities should be similar in the two regions. In contrast, the fraction of shocked molecular gas should be $\sim 5-7$ times larger in the spiral arm (up to $\sim 10 \%$ of the available molecular gas mass over the arm region) compared to the nuclear ring. In the light of these results, we revise the validity of the various scenarios that have been proposed to explain the onset of shock chemistry in galaxies and study their applicability to the nucleus of IC 342. We conclude that the large-scale shocks revealed by the SiO map of IC 342 are mostly unrelated to star formation and arise instead in a pre-starburst phase. Shocks are driven by cloud-cloud collisions along the potential well of the IC 342 bar. The general implications for the current understanding of galaxy evolution are discussed.
\end{abstract}

Key words. galaxies: individual: IC 342 - galaxies: starburst - galaxies: nuclei - ISM: molecules - molecular processes radio lines: galaxies

\section{Introduction}

There is mounting evidence that the properties of molecular gas in starbursts (SBs) and Active Galactic Nuclei (AGNs) differ from those of quiescent star forming galaxies (e.g., Genzel et al. 1998). The spectacular energies injected into the gas reservoirs of active galaxies can create a particularly harsh environment for the neutral ISM. Although thus far restricted

^ Based on observations carried out with the IRAM Plateau de Bure Interferometer. IRAM is supported by INSU/CNRS (France), MPG (Germany) and IGN (Spain). to a handful of objects, multiline millimeter wave studies have made possible to study the onset of large-scale shocks, the propagation of chemistry of Photon Dominated Regions (PDRs) or the prevalence of X-ray Dominated Regions (XDRs) in the molecular disks of SBs and AGNs (e.g., Mauersberger \& Henkel 1993; Tacconi et al. 1994; García-Burillo et al. 2000, 2001b, 2002; Martín et al. 2003, 2005; Usero et al. 2004; Fuente et al. 2005a; Meier \& Turner 2005).

The first $\operatorname{SiO}(v=0, J=2-1)$ maps made with the Plateau de Bure Interferometer (PdBI) in the nuclei of the prototypical starbursts NGC 253 and M 82 have revealed the existence 
of large-scale molecular shocks in galaxy disks (García-Burillo et al. 2000, 2001b). Different scenarios have been proposed to account for the emission of $\mathrm{SiO}$ in our own Galaxy and in the nuclei of external galaxies. On small $\sim$ pc-scale, studies in the Galaxy disk show that the enhancement of $\mathrm{SiO}$ in the gas phase can be produced in the bipolar outflows of young stellar objects (YSOs), due to the sputtering of dust grains by shocks (Martín-Pintado et al. 1992; Schilke et al. 1997; Cesaroni et al. 1999). On larger scales, Martín-Pintado et al. (1997) reported the detection of a $\mathrm{SiO} \sim 150 \mathrm{pc}$ circumnuclear disk (CND) in the Galactic Center region. In this CND high fractional abundances of $\mathrm{SiO}$ are found in molecular clouds which are not actively forming stars, but where bar models for our Galaxy predict a high likelihood for cloud collisions (Hüttemeister et al. 1998). In M 82 virtually all of the $\mathrm{SiO}$ emission traces the diskhalo interface, where episodes of mass injection are building up the gaseous halo (García-Burillo et al. 2001b). García-Burillo et al. (2000) have discussed the role of bar resonances in inducing shocks in the $\sim 600$ pc CND of NGC 253 . However, the high-inclination of NGC 253 and the limited perspective of the Galactic Center region make the determination of bar resonance positions mostly dependent on kinematical models.

In this paper we study at high-resolution $\left(\sim 5^{\prime \prime}\right)$ the $\mathrm{SiO}(v=$ $0, J=2-1)$ emission in the inner $r \sim 30^{\prime \prime}$ of IC 342, using the IRAM Plateau de Bure Interferometer (PdBI). IC 342 is a nearby ( $D \simeq 3.3 \mathrm{Mpc}$, i.e., $1^{\prime \prime} \simeq 16 \mathrm{pc}$; Saha et al. 2002) weakly barred spiral galaxy that hosts a moderate starburst episode in the central $r \sim 80$ pc nuclear region (Böker et al. 1997, 1999). Thanks to its nearly face-on orientation and close distance, IC 342 is an optimal testbed where the underlying mechanisms of large-scale molecular shocks can be probed using the PdBI. At this distance, the PdBI can trace and spatially resolve the $\mathrm{SiO}$ emission of shocked molecular gas on scales of individual GMCs $(\sim 80 \mathrm{pc})$ in the disk of IC 342. Several works have underlined the similarities between the nucleus of our Galaxy and that of IC 342 in terms of the measured gas mass fractions, stellar masses and star formation (Downes et al. 1992). This study can thus help to shed light on the origin of molecular shocks in the nucleus of our own Galaxy.

The distribution of molecular gas in the inner $r \sim 320 \mathrm{pc}$ of IC 342, revealed by the published interferometer CO maps of the galaxy, is reminiscent of the typical response of gas to a bar potential (Lo et al. 1984; Ishizuki et al. 1990; Levine et al. 1994; Meier \& Turner 2001, 2005; Schinnerer et al. 2003). Two gas lanes are shifted with respect to the major axis of the $\sim 9 \mathrm{kpc}$ bar oriented with a position angle $\sim 20^{\circ}$ (Buta \& McCall 1999). The gas lanes delineate a two-arm spiral pattern that ends at a nuclear $r \sim 80 \mathrm{pc}$ ring. This pattern would correspond to the transition from $x_{1}$ orbits (outer disk) to $x_{2}$ orbits (inner disk) of the IC 342 bar, assuming that an Inner Lindblad Resonance (ILR) exists near $r \sim 80-160 \mathrm{pc}$.

Previous interferometer maps have probed the dense gas content of the nucleus of IC 342 (Ho et al. 1990: $\mathrm{NH}_{3}$; Downes et al. 1992: HCN; Nguyen-Q-Rieu et al. 1992: $\mathrm{HCO}^{+}$; Meier \& Turner 2005: $\mathrm{HNC}, \mathrm{HC}_{3} \mathrm{~N}, \mathrm{C}_{2} \mathrm{H}, \mathrm{C}^{34} \mathrm{~S}, \mathrm{HNCO}, \mathrm{CH}_{3} \mathrm{OH}$ and $\mathrm{N}_{2} \mathrm{H}^{+}$). Five major GMCs, labelled originally as A-to-E in the HCN map of Downes et al. (1992) and later redefined by Meier $\&$ Turner (2001), are identified in the inner $r \sim 320$ pc disk.
Meier \& Turner (2005) find remarkable differences in morphology between the various molecular emission maps of IC 342 . This is interpreted as evidence of strong chemical differentiation in the nucleus of IC 342 . While some molecules trace the Photon Dominated Regions close to the nuclear starburst $(r \sim 80-180 \mathrm{pc})$, the emission of molecular species such as methanol $\left(\mathrm{CH}_{3} \mathrm{OH}\right)$ is considered to stem from shocks (Meier $\&$ Turner 2005).

In the scenario of shock chemistry there is evidence that $\mathrm{CH}_{3} \mathrm{OH}$ and $\mathrm{SiO}$ trace distinctly different velocity regimes in shocks. $\mathrm{SiO}$ is seen to be associated with more energetic events, i.e., those potentially more efficient in processing dust grains (Garay et al. 2000). In this paper we take advantage of the complementarity of $\mathrm{SiO}$ and $\mathrm{CH}_{3} \mathrm{OH}$ as tracers of shock chemistry and use the $\mathrm{SiO}$-to- $\mathrm{CH}_{3} \mathrm{OH}$ ratio in IC 342 to discuss the origin of large-scale shocks in this galaxy. With this aim we make a quantitative comparison of our results with those obtained in other well-known references for shock chemistry in our Galaxy and in external galaxies.

We describe in Sect. 2 the PdBI observations used in this paper. Section 3 presents the main results of the analysis of the continuum image and the $\mathrm{SiO}(2-1)$ and $\mathrm{H}^{13} \mathrm{CO}^{+}(1-0)$ line maps of IC 342. We derive in Sect. 4 the fractional abundances of $\mathrm{SiO}$ in the disk of the galaxy and interpret their relation to the published $\mathrm{CH}_{3} \mathrm{OH}$ map in Sect. 5. Section 6 discusses the possible mechanisms driving large-scale shocks in IC 342 . In Sect. 7 we analyze the potential role of density waves as drivers of the large-scale shock chemistry in IC 342. The main conclusions are summarized in Sect. 8.

\section{Observations}

Observations of IC 342 were carried out with the PdBI from July to August 2001. We observed simultaneously the $J=2-1$ line of $\mathrm{SiO}(86.847 \mathrm{GHz})$ and the $J=1-0$ line of $\mathrm{H}^{13} \mathrm{CO}^{+}$ $(86.754 \mathrm{GHz})$ using the $\mathrm{CD}$ set of configurations. The primary beam of the PdBI at $87 \mathrm{GHz}$ is $55^{\prime \prime}$. Two positions shifted $\left(0^{\prime \prime},-12^{\prime \prime}\right)$ and $\left(0^{\prime \prime},+12^{\prime \prime}\right)$ from the phase center, $\alpha_{\mathrm{J} 2000.0}=$ $03^{\mathrm{h}} 46^{\mathrm{m}} 48^{\mathrm{s}} .01, \delta_{\mathrm{J} 2000.0}=68^{\circ} 05^{\prime} 46^{\prime \prime} .0$, were observed in mosaic mode. We adjusted the spectral correlator to give a contiguous bandwidth of $1500 \mathrm{~km} \mathrm{~s}^{-1}$. The frequency resolution was set to $1.25 \mathrm{MHz}\left(4.3 \mathrm{~km} \mathrm{~s}^{-1}\right)$ during the observations; channels were resampled to a velocity resolution of $5 \mathrm{~km} \mathrm{~s}^{-1}$ in the final maps. We calibrated visibilities using $0224+671$ as amplitude and phase reference. The absolute flux scale was derived on MWC 349, and receiver passband was calibrated on 3C 454.3 and $3 \mathrm{C} 345$.

Mosaics were CLEANed using the MAPPING procedure of the GILDAS software package, which includes primary beam correction. The synthesized clean beam is 5 .' $6 \times 5$ ". 1 size $\left(\mathrm{PA}=321^{\circ}\right)$ for the line maps. Images are $300 \times 300$ pixels in extent, with a pixel size of 0.35 . The rms noise level in $5 \mathrm{~km} \mathrm{~s}^{-1}$ wide channels, derived after subtraction of the continuum emission, is $1.5 \mathrm{mJy}$ beam $^{-1}$ at the center of the maps.

A $3.5 \mathrm{~mm}$ continuum map was generated averaging channels free of line emission. Uniform weighting was applied to the measured visibilities, producing a clean beam of 4 '. $^{\prime} 1 \times 33^{\prime \prime} 8$ $\left(\mathrm{PA}=111^{\circ}\right)$. The rms at the center is $0.45 \mathrm{mJy} \mathrm{beam}^{-1}$. 


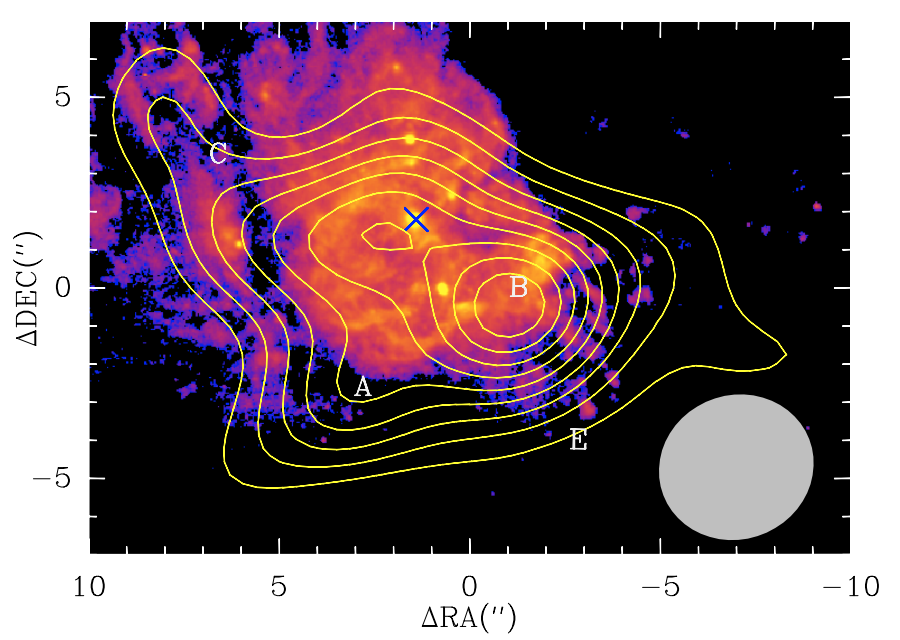

Fig. 1. We overlay the $3.5 \mathrm{~mm}$ continuum map obtained with the PdBI (contour levels: 0.90 to 4.5 in steps of $0.45 \mathrm{mJy}^{\text {beam }}{ }^{-1}$ ) with the $\mathrm{H} \alpha+$ continuum image (grey scale in arbitrary units) obtained by HST towards the nucleus of IC 342. Positions of GMCs A, B, C and E (as defined by Meier \& Turner 2001), are highlighted; the position of GMC D, beyond the displayed region of this figure, has been redefined in this work according to the local maximum of $\mathrm{SiO}$ integrated intensity (see Fig. 2). A white cross marks the position of the galactic center determined by Schinnerer et al. (2003). Relative positions are referred to the phase center $\alpha_{\mathrm{J} 2000.0}=03^{\mathrm{h}} 46^{\mathrm{m}} 48^{\mathrm{s}} .01, \delta_{\mathrm{J} 2000.0}=68^{\circ} 05^{\prime} 46^{\prime \prime} 0$. The 4 .' $1 \times 3$.' 8 beam is drawn at the bottom-right corner.

\section{Results}

\subsection{The $3.5 \mathrm{~mm}$ continuum map}

The $3.5 \mathrm{~mm}$ continuum emission contours are displayed in Fig. 1. The total flux integrated within the inner $r \sim 180 \mathrm{pc}$ of the galaxy is $\sim 17 \mathrm{mJy}$. The flux recovered is of $\sim 22 \mathrm{mJy}$ when derived from a lower 9 " resolution version of the PdBI map; the latter is obtained assuming a Gaussian UV taper on the visibilities (with a $F W H M=70 \mathrm{~m}$ ). These values are roughly in agreement with the previous estimates obtained by Downes et al. (1992) and Meier \& Turner (2001) at similar frequencies. The bulk of the continuum emission comes from the inner $r \sim 80$ pc of IC 342 and it is closely linked to the nuclear star forming region identified in the $\mathrm{H} \alpha+$ continuum HST image of the galaxy (Fig. 1). As illustrated in Fig. 2, the continuum emission is mostly anticorrelated with the emission coming from the dense molecular gas traced by $\mathrm{SiO}$ or $\mathrm{H}^{13} \mathrm{CO}^{+}$.

Despite its compactness, the continuum source is spatially resolved by the PdBI beam. The morphology of the emission follows closely the distribution of star forming complexes in the nucleus of IC 342: two 5 Myr-old H II complexes (Böker et al. 1997) which are close to GMCs B and C (notation of Meier \& Turner 2001), and an older (6-60 Myr) star cluster close to the center of the galaxy (Böker et al. 1997, 1999). As shown in Fig. 1, two peaks of emission shape the morphology of an elongated disk oriented along $\mathrm{PA} \simeq 64^{\circ}$. The principal peak is related to the western H II complex, which is identified in the HST map to be close to GMC B. The secondary maximum is close to the dynamical center, where the old star cluster is detected. Lower-level emission extends East from the central disk towards GMCs A and C. The latter extension is close to the eastern H II complex.

Accounting for the differences in spatial resolution and sensitivity, the morphology of the $3.5 \mathrm{~mm}$ continuum PdBI map agrees with that of previous radiocontinuum maps obtained at other wavelengths (Condon et al. 1982: $21 \mathrm{~cm}$; Turner \& Ho 1983: 2 and $6 \mathrm{~cm}$; Ho et al. 1990: $1.3 \mathrm{~cm}$; Downes et al. 1992: $3.4 \mathrm{~mm})$. At higher frequencies, however, the contribution from dust to the thermal emission may not be negligible. This would explain the differences between the continuum map of Fig. 1 and the $1.3 \mathrm{~mm}$ continuum image obtained by Meier \& Turner (2001).

Based on measurements at various frequencies, previous works have concluded that thermal free-free bremsstrahlung should dominate the emission budget at $3.5 \mathrm{~mm}$ (Downes et al. 1992; Turner \& Ho 1983; Turner \& Hurt 1992). The good spatial coincidence between the $3.5 \mathrm{~mm}$ and the $\mathrm{H} \alpha$ emissions supports the conclusion that the $3.5 \mathrm{~mm}$ emission traces the location of ongoing star formation in the inner $r \sim 80$ pc of IC 342.

\subsection{The line maps: $\mathrm{SiO}$ and $\mathrm{H}^{13} \mathrm{CO}^{+}$}

\subsubsection{Integrated intensity maps and line ratios}

Figure 2 shows the velocity-integrated intensity maps of $\mathrm{SiO}(2-1)$ and $\mathrm{H}^{13} \mathrm{CO}^{+}(1-0)$ in the inner $r \sim 320 \mathrm{pc}$ of IC 342 . The spatial resolution of the PdBI map allows us to resolve the overall emission which, for both tracers, is elongated along the N-S direction. Though with significant differences between $\mathrm{SiO}$ and $\mathrm{H}^{13} \mathrm{CO}^{+}$, the basic morphology of the maps is roughly in agreement with that of the HCN map of Downes et al. (1992). In the case of $\mathrm{SiO}$, we detect strong emission in the spiral arm located to the North (GMCs C and D in Fig. 2). Weaker $\mathrm{SiO}$ emission delineates the southern ridge of the nuclear ring (GMCs A and E in Fig. 2). Finally, SiO emission is not detected over the southern spiral arm. This result agrees with the overall picture derived from other dense gas tracers in IC 342 which are hardly detected over the southern spiral. Compared to $\mathrm{SiO}$, the emission of $\mathrm{H}^{13} \mathrm{CO}^{+}$is weaker along the northern spiral arm, while the peak of emission is found on the southern ridge of the nuclear ring.

As shown in Fig. 3, the different distributions of $\mathrm{SiO}$ and $\mathrm{H}^{13} \mathrm{CO}^{+}$in IC 342 translate into an order of magnitude difference in the $\mathrm{SiO}$-to- $\mathrm{H}^{13} \mathrm{CO}^{+}$intensity ratio, $R_{I}$, which goes from $\sim 3.3$ on the northern spiral arm down to $\sim 0.3$ on the nuclear ring. The average value of $R_{I}$ inside the image field of view is $\sim 1.6$. If we assume that the emission of both lines is optically thin, the value of $R_{I}$ provides an estimate of the beam-averaged fractional abundance of $\mathrm{SiO}$ relative to $\mathrm{H}^{13} \mathrm{CO}^{+}$, which is accurate within a factor of 4 (see Sect. 4 for discussion). The reference studies of galactic clouds (Martín-Pintado et al. 1992; Bachiller \& Pérez-Gutiérrez 1997; Fuente et al. 2005b) and external galaxies (García-Burillo et al. 2000, 2001b; Usero et al. 2004) indicate that a value of $R_{I}>0.1$ is a strong indication that shock chemistry is at work in molecular gas. The reported values of $R_{I}$ for IC 342 are similar to those found on similar spatial scales in the circumnuclear disk of NGC 253 ( 1-3; García-Burillo et al. 2000) and, also, in the 

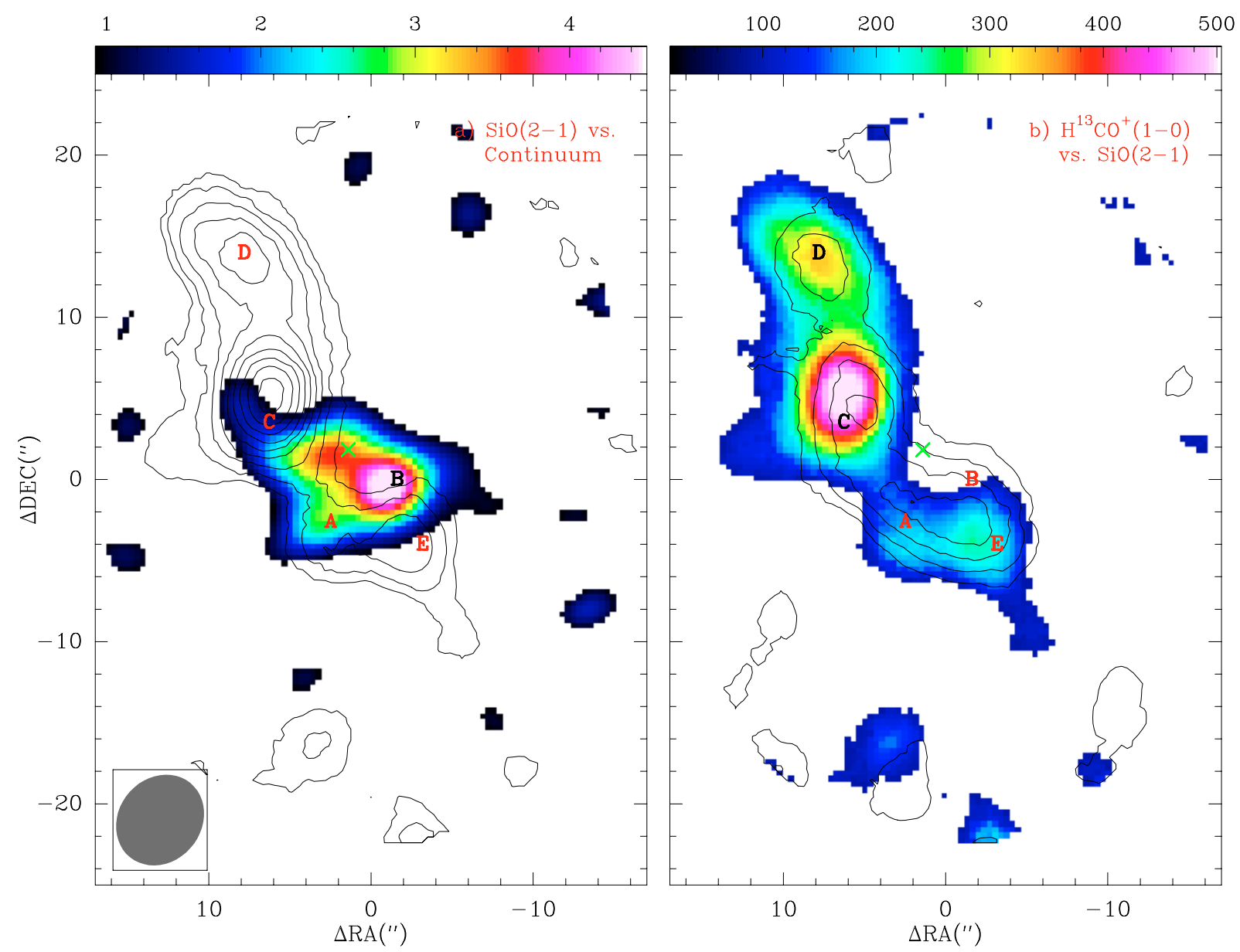

Fig. 2. a) (Left) We overlay the $\mathrm{SiO}(2-1)$ integrated intensity map (contour levels from 90 to 522 in steps of $54 \mathrm{mJy} \mathrm{beam}^{-1} \mathrm{~km} \mathrm{~s}^{-1} ; 1 \sigma=$ $36 \mathrm{mJy}$ beam ${ }^{-1} \mathrm{~km} \mathrm{~s}^{-1}$ ) with the $3.5 \mathrm{~mm}$ continuum map (grey scale from 0.90 to $4.5 \mathrm{mJy}$ beam $^{-1} ; 1 \sigma=0.45 \mathrm{mJy} \mathrm{beam}^{-1}$ ). b) $($ Right ) Same as a) with $\mathrm{H}^{13} \mathrm{CO}^{+}(1-0)$ (contours) overlaid with $\mathrm{SiO}(2-1)$ (grey scale), both displayed with the same intensity spacing (used in a)). The two integrated emission maps were obtained with a $1.2 \sigma$ clipping applied to the velocity interval $[-10,85] \mathrm{km} \mathrm{s}^{-1}$. Line contours are screened beyond the displayed field-of-view as $\mathrm{S} / \mathrm{N}$ ratio is lower than 2.5 farther out. Beam-size is represented by a filled ellipse.

chimney and the supershell of M $82(\sim 0.4-3.5$; García-Burillo et al. 2001b).

Of particular note, $R_{I}$ reaches the largest values along the northern spiral arm, i.e., in a region of the disk of IC 342 where the evidence of active star formation is scarce. Regardless of its origin, the order of magnitude variation of $R_{I}$ measured over the disk of IC 342 reveals that shocks are processing molecular gas with a highly changing efficiency (in terms of the total mass of grain material processed by shocks relative to the total gas mass; see discussion in Sects. 4 and 5).

\subsubsection{Gas kinematics and line profiles}

Figure 4 shows the velocity-channel maps of $\mathrm{SiO}(2-1)$ and $\mathrm{H}^{13} \mathrm{CO}^{+}(1-0)$ in the nucleus of IC 342 . For both tracers we see the expected velocity gradient due to the rotation of the disk, which according to the fit of Crosthwaite et al. (2001) should be maximal along the kinematic major axis at $\mathrm{PA}=37^{\circ}$. The channel maps show that the gas kinematics are similar for $\mathrm{SiO}$ and $\mathrm{H}^{13} \mathrm{CO}^{+}$on the nuclear ring: the velocity centroids and linewidths measured in $\mathrm{SiO}$ and $\mathrm{H}^{13} \mathrm{CO}^{+}$are roughly in agreement in this region (GMCs $\mathrm{A}, \mathrm{B}$, and $\mathrm{E}$ ). However, the gas kinematics show significant differences between the two species over the northern spiral arm (GMCs C and D): the emission of $\mathrm{SiO}$ is detected from $v=16 \mathrm{~km} \mathrm{~s}^{-1}$ to $v=66 \mathrm{~km} \mathrm{~s}^{-1}$ near GMC D, i.e., roughly twice the corresponding velocity interval for $\mathrm{H}^{13} \mathrm{CO}^{+}$.

The different kinematics of the $\mathrm{SiO}$ and $\mathrm{H}^{13} \mathrm{CO}^{+}$lines over the northern spiral arm are illustrated by the position-velocity plots shown in Fig. 5. SiO lines become significantly wider than $\mathrm{H}^{13} \mathrm{CO}^{+}$lines at the passage of the northern spiral arm GMCs (C and D), in contrast with the nuclear ring GMCs (B and E) where linewidths are the same within the errors. While the velocity centroids derived in the two lines are similar in the spiral arm region, the measured linewidths for $\mathrm{SiO}$ are, on average, a factor of 2 larger than the linewidths of $\mathrm{H}^{13} \mathrm{CO}^{+}$. The estimated velocity dispersion of the GMCs $\left(\sigma_{v}\right)$ is $\sim 20-25 \mathrm{~km} \mathrm{~s}^{-1}$, in the northern spiral arm, and $\sim 10 \mathrm{~km} \mathrm{~s}^{-1}$, in the nuclear ring. This suggests that the degree of apparent turbulence measured on GMC-like scales in the $\mathrm{SiO}$ emitting gas is enhanced compared to that of the more quiescent dense gas component traced by $\mathrm{H}^{13} \mathrm{CO}^{+}$in this region. The latter implies that the $\mathrm{SiO}(2-1)$-to- $\mathrm{H}^{13} \mathrm{CO}^{+}(1-0)$ ratio measured at the wings of the $\mathrm{SiO}$ lines should be even larger than the 


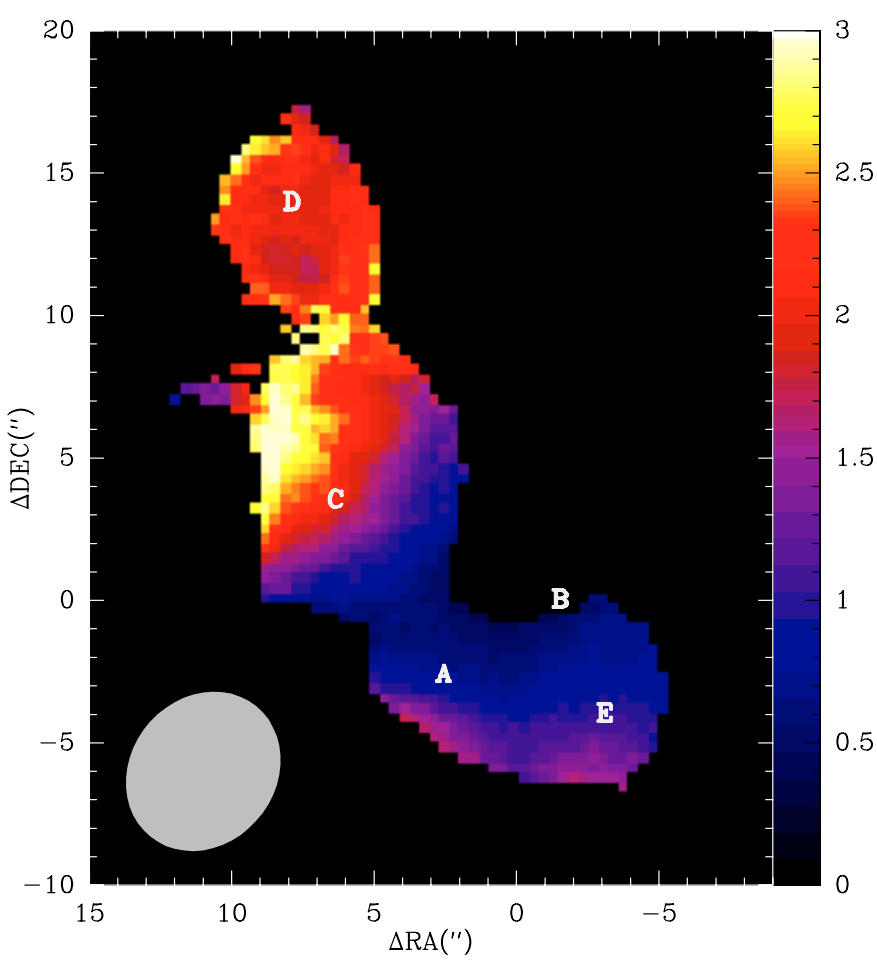

Fig. 3. $\mathrm{SiO}(2-1)$-to- $\mathrm{H}^{13} \mathrm{CO}^{+}(1-0)$ integrated intensity ratio in the inner $r \sim 320 \mathrm{pc}$ disk of IC 342. The ratio is derived from the two maps of Fig. 2, assuming a $2.5 \sigma$-clipping on the integrated intensities of both lines. The measured ratios (grey scale) range from 0.3 to 3.3 .

velocity-integrated ratio $R_{I}$ derived above for the northern spiral arm. This would only reinforce the case of an enhanced $\mathrm{SiO}$ chemistry in gas phase in this region.

Figure 6 shows the $\mathrm{SiO}$ and $\mathrm{H}^{13} \mathrm{CO}^{+}$spectra observed towards the positions of GMCs A-to-E (the corresponding Gaussian fits are listed in Table 1). As sketched in Fig. 6, we report on the tentative detection of two out of the four hyperfine line components of the $N_{K^{-} K^{+}}=1-0$ group of transitions of $\mathrm{HCO}$ towards the position of GMC C. These correspond to the rest frequencies $86.671 \mathrm{GHz}(J=3 / 2-1 / 2, F=2-1)$ and $86.777 \mathrm{GHz}(J=1 / 2-1 / 2, F=1-1)$. The HCO $(F=2-1)$-to$\mathrm{H}^{13} \mathrm{CO}^{+}(1-0)$ intensity ratio inferred at GMC C is $\sim 0.51 \pm 0.16$; comparable ratios $(\sim 0.15-0.5)$ were derived by García-Burillo et al. (2002) for the $\sim 650 \mathrm{pc}$ nuclear disk of $\mathrm{M} 82$, where the large $\mathrm{HCO}$ abundances $\left(X(\mathrm{HCO}) \sim 4 \times 10^{-10}\right)$ indicate that the whole inner disk can be viewed as a giant PDR. The tentative detection of $\mathrm{HCO}$ towards $\mathrm{C}$ would be well accounted for if UV fields are partly driving the chemistry of the molecular clouds closest to the embedded star forming complex identified in the NIR by Böker et al. (1997; see García-Burillo et al. 2002, for a discussion on the chemistry of the HCO molecule).

\section{SiO fractional abundances}

We have estimated the column densities $(N)$ of $\mathrm{SiO}$ and $\mathrm{H}^{13} \mathrm{CO}^{+}$from the PdBI maps of IC 342 using a Large Velocity Gradient (LVG) code. Our aim is to estimate the abundance of $\mathrm{SiO}$ relative to $\mathrm{H}_{2}(X(\mathrm{SiO}))$ inferred here from the SiOto- $\mathrm{H}^{13} \mathrm{CO}^{+}$column density ratio $\left(R_{N}\right)$. Values of $N(\mathrm{SiO})$ and $N\left(\mathrm{H}^{13} \mathrm{CO}^{+}\right)$are derived from measured integrated intensities, assuming a plausible range of physical conditions for the gas. Given that the emission of both species is optically thin, $R_{N}$ is proportional to the $\mathrm{SiO} / \mathrm{H}^{13} \mathrm{CO}^{+}$intensity ratio $\left(R_{I}\right)$. Since $\mathrm{SiO}$ and $\mathrm{H}^{13} \mathrm{CO}^{+}$have similar dipole moments and the observed transitions have comparable upper state energies, it is reasonable to assume the same physical conditions for both species. We have run five LVG models covering a range of gas densities $\left(n\left(\mathrm{H}_{2}\right)\right)$ from $10^{4} \mathrm{~cm}^{-3}$ to $10^{6} \mathrm{~cm}^{-3}$. The explored interval encompasses the total range of molecular gas densities determined in the GMCs of IC 342 from multitransition studies of CO and HCN (e.g., see Schulz et al. 2001). As gas kinetic temperature, we adopt a value $T_{\mathrm{K}}=50 \mathrm{~K}$ (Downes et al. 1992). The value of $T_{\mathrm{K}}$ is not critical in the estimate of $R_{N}$ within the range of $n\left(\mathrm{H}_{2}\right)$ explored in these calculations: the inferred column density ratios are similar within the temperature interval $20 \mathrm{~K} \leq T_{\mathrm{K}} \leq 80 \mathrm{~K}$ and $R_{N}$ increases, at most, by $40 \%$ if $T_{\mathrm{K}}$ is lowered to $10 \mathrm{~K}$.

We show in Fig. 7 the values estimated for $R_{N}$ towards GMCs A-to-E for $T_{\mathrm{K}}=50 \mathrm{~K}$ particularized for the different $n\left(\mathrm{H}_{2}\right)$ values. On average, $R_{N}$ is seen to increase by a factor of $\sim 4$ when $n\left(\mathrm{H}_{2}\right)$ is lowered from $10^{6} \mathrm{~cm}^{-3}$ to $10^{4} \mathrm{~cm}^{-3}$. The fractional abundance of $\mathrm{SiO}$ is inferred from $R_{N}$ assuming a standard abundance for $\mathrm{H}^{13} \mathrm{CO}^{+}$. The assumption of a standard value for $X\left(\mathrm{H}^{13} \mathrm{CO}^{+}\right)$is supported by observations of molecular clouds in our Galaxy. Contrary to $\mathrm{SiO}$, for which measured abundances are seen to differ by several orders of magnitude between quiescent clouds and shocked regions, $\mathrm{H}^{13} \mathrm{CO}^{+}$ shows a fairly stable abundance in a large variety of physical and chemical environments (see discussion in García-Burillo et al. 2000). Here we adopt $X\left(\mathrm{H}^{13} \mathrm{CO}^{+}\right)=2.5 \times 10^{-10}$; this corresponds to a typical abundance of the main isotope $X\left(\mathrm{H}^{12} \mathrm{CO}^{+}\right)=10^{-8}$ and to an isotopic ratio $\left[{ }^{12} \mathrm{C}\right] /\left[{ }^{13} \mathrm{C}\right] \simeq 40$ (Henkel et al. 1998).

The estimated abundance of $\mathrm{SiO}$ is $>2 \times 10^{-10}$ for all GMCs, i.e., at least two orders of magnitude larger than the typical $\mathrm{SiO}$ abundances of Galactic quiescent clouds (Martín-Pintado et al. 1992). This lower limit on the beam-averaged value of $X(\mathrm{SiO})$ measured here on GMC-like scales $(\sim 80 \mathrm{pc})$ indicates that shock chemistry is at work in the inner $r \sim 320$ pc disk of IC 342. Most remarkably, we see an overall N-S gradient in the value estimated for $X(\mathrm{SiO})$. On average, the abundance of $\mathrm{SiO}$ is nearly one order of magnitude larger in the northern spiral arm (e.g., $\sim 1-4 \times 10^{-9}$ at GMC D) than in the nuclear ring (e.g., $\sim 2-7 \times 10^{-10}$ at GMC B) within the explored range of densities. Schulz et al. (2001) have estimated the average densities of GMCs A-to-E based on a multitransition study of $\mathrm{HCN}$ and their isotopes. The results of this study indicate that average densities are a factor of 2 larger in the nuclear ring GMCs compared to those measured in the northern spiral arm. This would imply that the $\mathrm{N}-\mathrm{S}$ gradient on the value of $X(\mathrm{SiO})$ may be even larger than estimated above. Moreover, the overall mass budget of IC 342 is seen to be heavily weighted by molecular gas with typical densities $\sim 10^{3}-10^{4} \mathrm{~cm}^{-3}$ (Downes et al. 1992; Israel \& Baas 2003). Should this diffuse gas partly contribute to the emission of both species, this would imply that the $\mathrm{SiO}$ abundances may have to be boosted for all the GMCs (see Fig. 7) compared to the values reported above, thus reinforcing the case for shock chemistry. 


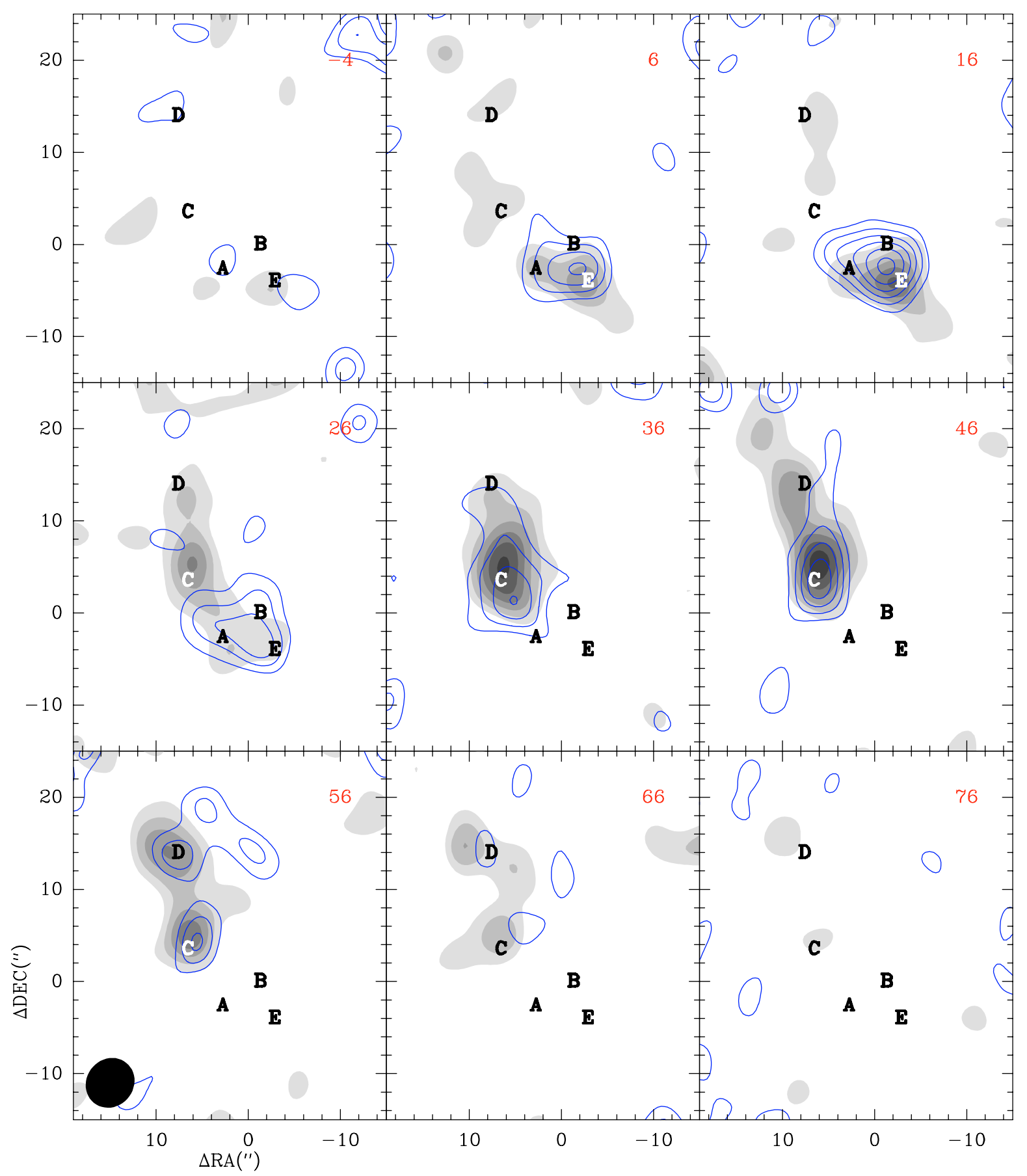

Fig. 4. Velocity channel maps of $\mathrm{SiO}(2-1)$ (grey scale) and $\mathrm{H}^{13} \mathrm{CO}^{+}(1-0)$ (contour levels) in $\mathrm{IC} 342$. Both scales are from $2.5 \sigma$ to $10.5 \sigma$ in steps of $1.5 \sigma ; 1 \sigma=1.1 \mathrm{mJy}^{-1}$ beam ${ }^{-1}$ in a $10 \mathrm{~km} \mathrm{~s}^{-1}$ channel. The channel LSR-velocities and the positions of GMCs A-to-E are indicated in each panel.

The beam-averaged values of $X(\mathrm{SiO})$ derived above are necessarily lower limits to the real abundance attained by $\mathrm{SiO}$ in the fraction of dense molecular gas which is being processed by shocks in IC 342 (hereafter $\left.X(\mathrm{SiO})\right|_{\text {shock }}$ ). If we denote by $f_{\text {shock }}$ the a priori unknown fraction of shocked dense gas, $X(\mathrm{SiO})$ can be formally factorized as:

$X(\mathrm{SiO})=f_{\text {shock }} \times\left. X(\mathrm{SiO})\right|_{\text {shock }}$.

The notable N-S gradient found in the derived value of $X(\mathrm{SiO})$ between the nuclear ring and the northern spiral arm could be accounted by two extreme scenarios: first, an order of magnitude change in $f_{\text {shock }}$ between the two regions, or alternatively, a similar change in $\left.X(\mathrm{SiO})\right|_{\text {shock}}$. While the first scenario would imply that the typical column densities of shocked gas in the northern spiral arm are $~ 6-7$ times larger than in the nuclear ring, the second scenario would call for a notably different shock velocity regime $\left(v_{\text {shock }}\right)$ in the two regions. In this case $v_{\text {shock }}$ should be significantly larger in the northern spiral arm. As is discussed in Sect. 7, finding the right scenario between the two proposed above is key to shed light on the nature of 


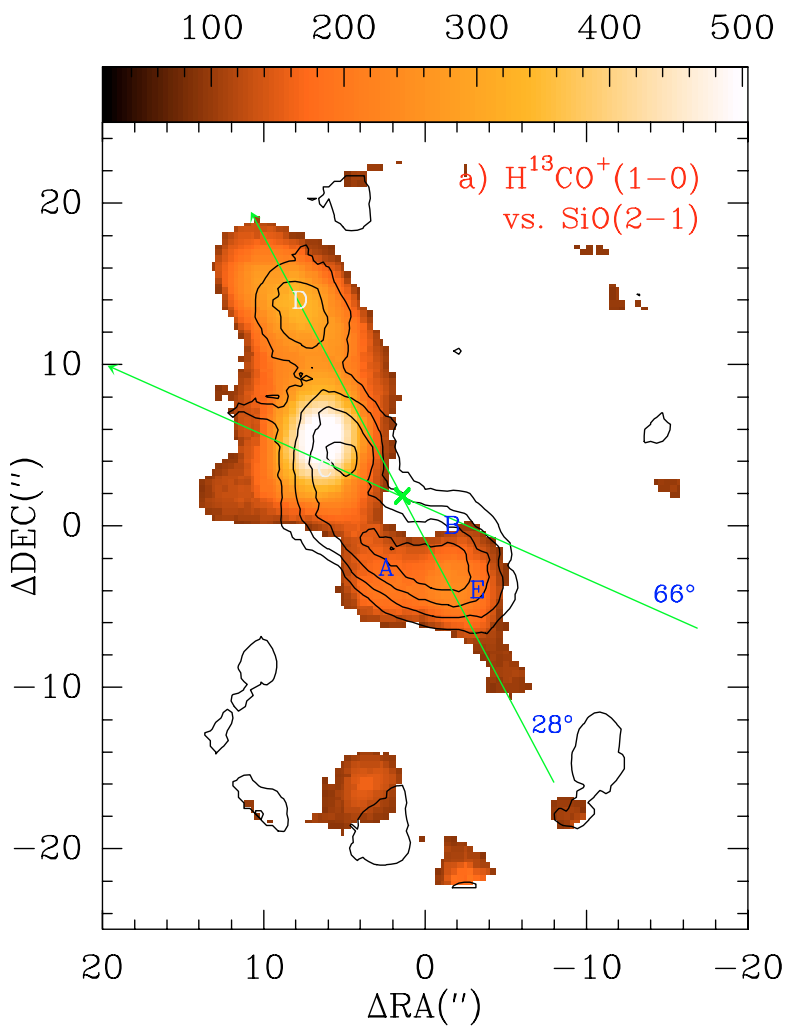

0

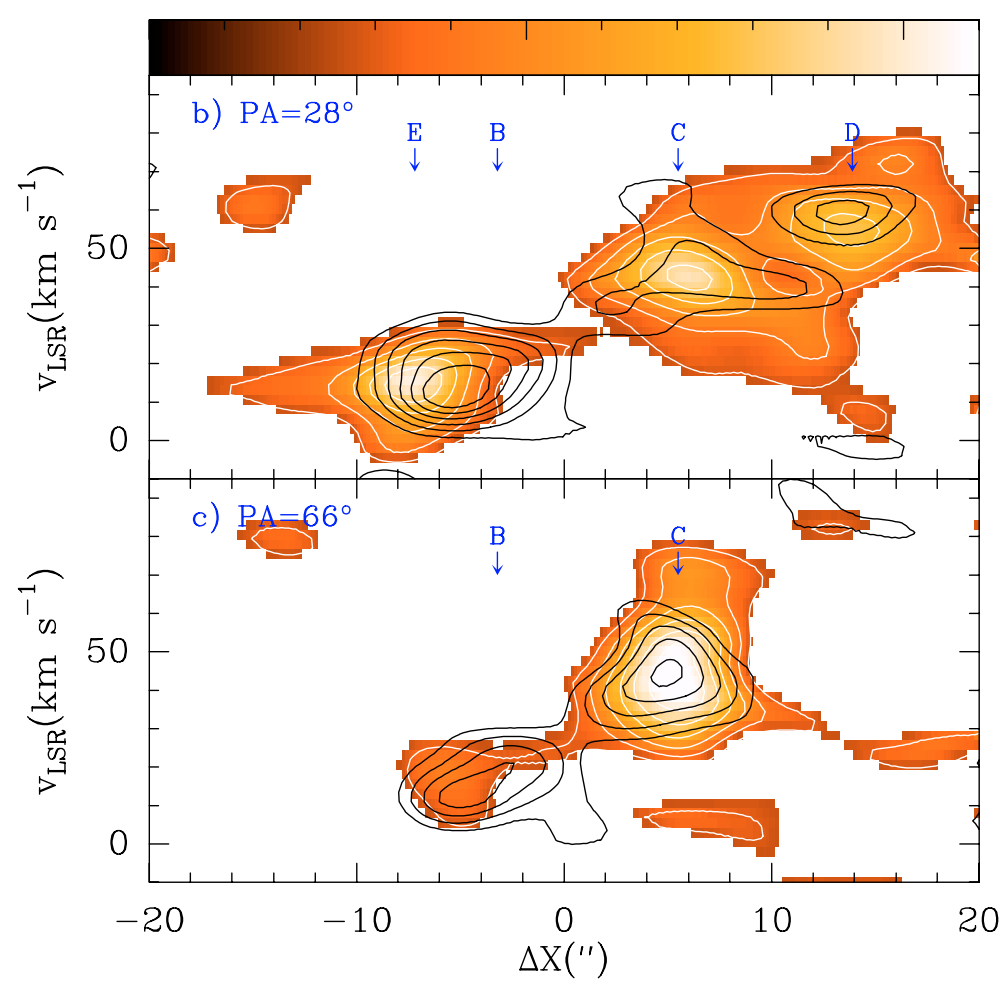

Fig. 5. $\mathrm{SiO}(2-1)$ and $\mathrm{H}^{13} \mathrm{CO}^{+}(1-0)$ position-velocity $(p-v)$ diagrams (b) (top right) and c) (bottom right)) taken along the $1 \mathrm{D}$-strips highlighted in a) (left) (overlay of the $\mathrm{SiO}(2-1)$ and $\mathrm{H}^{13} \mathrm{CO}^{+}(1-0)$ intensity maps as shown in Fig. 2b). The position angles of the $p-v$ diagrams are chosen to maximize the contrast between the linewidths measured in the northern spiral arm and in the nuclear ring. In the two $p-v$ plots, the $\mathrm{SiO}(2-1)$ brightness is represented in grey scale $\left(2.6\right.$ to $11 \mathrm{mJy}_{\text {beam }}^{-1}$ ) and white contours (from 3 to 11 in steps of $\left.1.5 \mathrm{mJy} \mathrm{beam}^{-1}\right)$, while $\mathrm{H}^{13} \mathrm{CO}^{+}(1-0)$ brightness levels appear in black contours (same levels as above). The approximate location of GMCs B,C, D and E along the strips is indicated in b) and c).
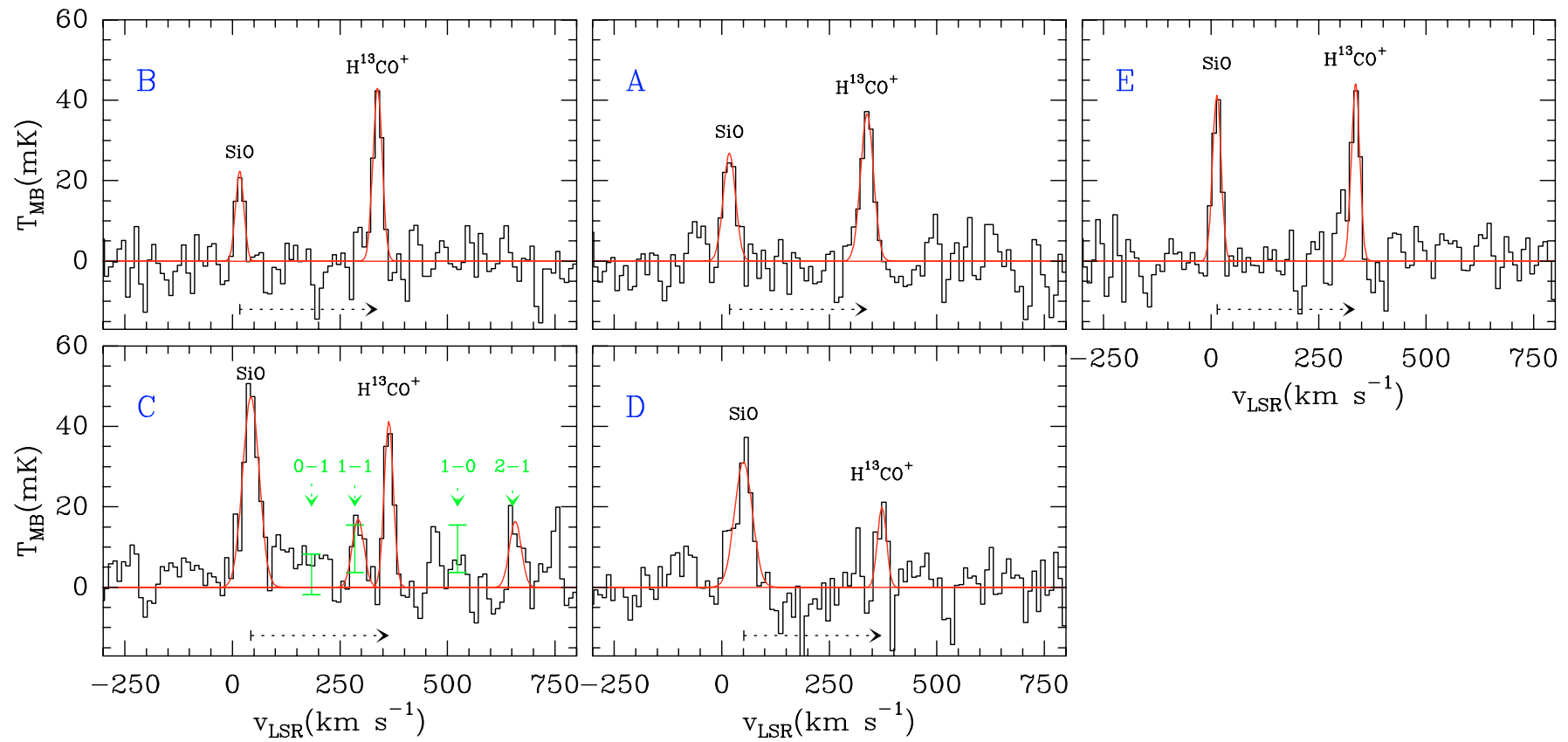

Fig. 6. $\mathrm{SiO}(2-1)$ and $\mathrm{H}^{13} \mathrm{CO}^{+}(1-0)$ spectra observed towards GMCs A-to-E. Spectra are smoothed to $10 \mathrm{~km} \mathrm{~s}^{-1}$ resolution. LSR-velocities are referred to the frequency of $\mathrm{SiO}(2-1)$. A horizontal arrow marks the expected velocity of $\mathrm{H}^{13} \mathrm{CO}^{+}(1-0)$ at each panel according to the measured centroid for the $\mathrm{SiO}(2-1)$ line (i.e., redshifted $\sim+321 \mathrm{~km} \mathrm{~s}^{-1}$ from the $\mathrm{SiO}$ line). The expected velocities for the four hyperfine lines of $\mathrm{HCO}$ are marked by vertical arrows on the panel of GMC C. Vertical $\pm 1 \sigma$ errorbars indicate the expected peak temperatures of the HCO hyperfine lines, fixing the $\operatorname{HCO}(J=3 / 2-1 / 2 ; F=2-1)$ temperature from observations and assuming optically thin emission for the four lines. 
Table 1. Parameters of Gaussian fits of the $\mathrm{SiO}(2-1), \mathrm{H}^{13} \mathrm{CO}^{+}(1-0)$ and $\mathrm{HCO}(1-0)$ lines of Fig. 6. Errors (in brackets) are $1 \sigma$ values.

\begin{tabular}{|c|c|c|c|c|c|}
\hline & (1) & (2) & (3) & (4) & (5) \\
\hline & Line & $\begin{array}{l}T_{\text {peak }} \\
(\mathrm{mK})\end{array}$ & $\begin{array}{c}I \\
\left(\mathrm{~K} \mathrm{~km} \mathrm{~s}^{-1}\right) \\
\end{array}$ & $\begin{array}{c}v_{\mathrm{LSR}} \\
\left(\mathrm{km} \mathrm{s}^{-1}\right)\end{array}$ & $\begin{array}{c}\Delta v_{1 / 2} \\
\left(\mathrm{~km} \mathrm{~s}^{-1}\right)\end{array}$ \\
\hline \multirow[t]{2}{*}{ A } & $\mathrm{SiO}(2-1)$ & $27(6)$ & $0.96(0.15)$ & $18(5)$ & $34(6)$ \\
\hline & $\mathrm{H}^{13} \mathrm{CO}^{+}(1-0)$ & $37(6)$ & $1.37(0.16)$ & $17 \quad(5)$ & $35 \quad(5)$ \\
\hline \multirow[t]{2}{*}{ B } & $\mathrm{SiO}(2-1)$ & $22(5)$ & $0.54(0.10)$ & $17 \quad(5)$ & $23(5)$ \\
\hline & $\mathrm{H}^{13} \mathrm{CO}^{+}(1-0)$ & $43 \quad(5)$ & $1.15(0.12)$ & $17 \quad(5)$ & $25(5)$ \\
\hline \multirow[t]{4}{*}{$\mathrm{C}$} & $\mathrm{SiO}(2-1)$ & $48(5)$ & $2.26(0.21)$ & $43 \quad(5)$ & $45(6)$ \\
\hline & $\mathrm{H}^{13} \mathrm{CO}^{+}(1-0)$ & $42(5)$ & $1.10(0.14)$ & $42(5)$ & $25(5)$ \\
\hline & $\mathrm{HCO}(\mathrm{F}=1-1)$ & $17(5)$ & $0.58(0.16)$ & $50(5)$ & $32(9)$ \\
\hline & $\operatorname{HCO}(\mathrm{F}=2-1)$ & $16(5)$ & $0.56(0.16)$ & $49 \quad(6)$ & $32(9)$ \\
\hline \multirow[t]{2}{*}{$\mathrm{D}$} & $\overline{\mathrm{SiO}(2-1)}$ & $31 \quad(5)$ & $1.61(0.20)$ & $\begin{array}{ll}51 \quad(5) \\
\end{array}$ & $48 \quad(8)$ \\
\hline & $\underline{\mathrm{H}^{13} \mathrm{CO}^{+}(1-0)}$ & $20(5)$ & $0.52(0.12)$ & $52 \quad(5)$ & $24(5)$ \\
\hline \multirow[t]{2}{*}{$\mathrm{E}$} & $\mathrm{SiO}(2-1)$ & $42(6)$ & $1.03(0.11)$ & $13(5)$ & $23(5)$ \\
\hline & $\mathrm{H}^{13} \mathrm{CO}^{+}(1-0)$ & $44(6)$ & $1.05(0.12)$ & $15(5)$ & $22(5)$ \\
\hline
\end{tabular}

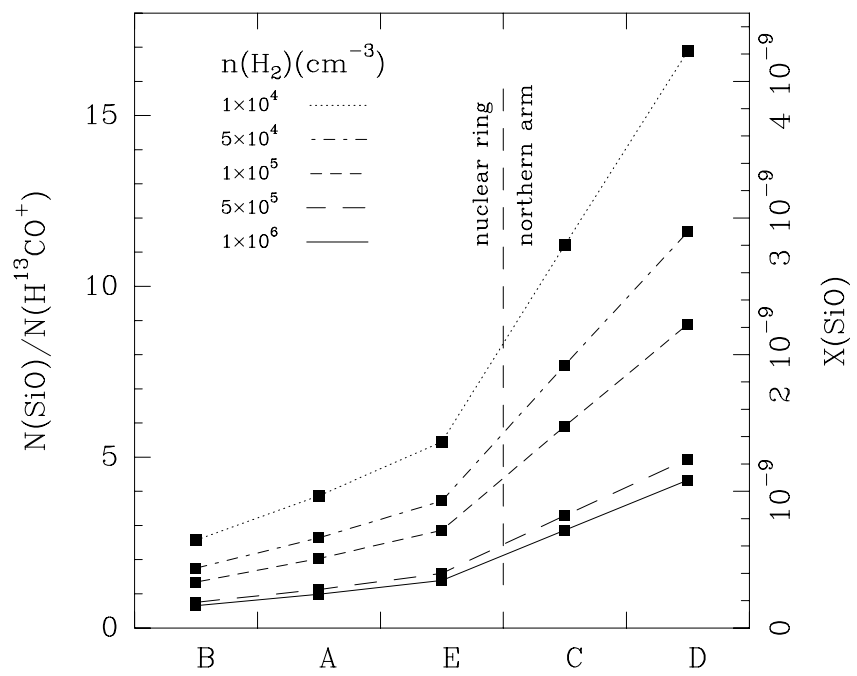

Fig. 7. $\mathrm{SiO}-$ to- $\mathrm{H}^{13} \mathrm{CO}^{+}$column density ratios and $\mathrm{SiO}$ abundances in GMCs A-to-E derived from a LVG calculation, assuming $T_{\mathrm{K}}=50 \mathrm{~K}$ and five different values of $n\left(\mathrm{H}_{2}\right)$ from $10^{4}$ to $10^{6} \mathrm{~cm}^{-3}$. GMCs are ordered along the $\mathrm{X}$-axis by increasing $X(\mathrm{SiO})$.

the driving mechanism of shocks in IC 342. In Sect. 5 we compare the emission of $\mathrm{SiO}$ in IC 342 with that of $\mathrm{CH}_{3} \mathrm{OH}$, another molecular shock tracer. In particular, we use the SiO-to$\mathrm{CH}_{3} \mathrm{OH}$ ratio derived for IC 342 to explore the origin of shocks in this galaxy.

\section{Molecular shock chemistry in IC 342}

\subsection{Tracers of shocks in molecular gas}

The significant enhancement of $\mathrm{SiO}$ in the gas phase is considered to be an indication that shock chemistry is at work in molecular gas (Martín-Pintado et al. 1992). The injection of Si-bearing material from dust grains into the gas phase, either through sputtering or grain-grain collisions, can explain the measured abundances of this molecule in shocked regions (Schilke et al. 1997; Caselli et al. 1997). Shocks are often

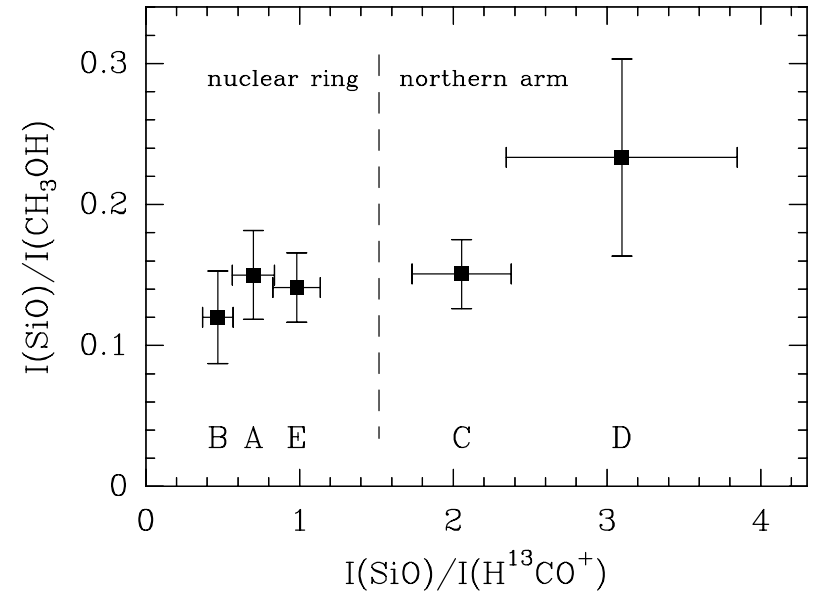

Fig. 8. We represent the $\mathrm{SiO}-$ to- $\mathrm{CH}_{3} \mathrm{OH}$ integrated intensity ratio as a function of the SiO-to- $\mathrm{H}^{13} \mathrm{CO}^{+}$ratio $\left(R_{I}\right)$ in GMCs A-to-E. $\mathrm{CH}_{3} \mathrm{OH}$ data have been taken from Meier \& Turner (2005).

invoked to account for the large abundances of other molecular species measured in bipolar outflows. This is the case of $\mathrm{CH}_{3} \mathrm{OH}$ (Bachiller et al. 1995). Although qualitatively similar, shocks characterized by different velocity regimes are expected to process to a different extent dust grains in molecular gas. Fast shocks $\left(v_{\text {shock }}>15-20 \mathrm{~km} \mathrm{~s}^{-1}\right)$ can destroy the grain cores, liberating refractory elements to the gas phase (Schilke et al. 1997; Caselli et al. 1997). In contrast, while slow shocks $\left(v_{\text {shock }}<10-15 \mathrm{~km} \mathrm{~s}^{-1}\right)$ are not able to destroy the grain cores, they can heavily process the icy grain mantles. The assumed different location of Si-bearing material (cores) and solid-phase $\mathrm{CH}_{3} \mathrm{OH}$ (mantles) in dust grains makes of $\mathrm{SiO}$ and $\mathrm{CH}_{3} \mathrm{OH}$ good tracers of fast and slow shocks, respectively. Furthermore, for velocities above $\sim 10-15 \mathrm{~km} \mathrm{~s}^{-1}$ shocks could destroy the molecules in gas-phase of volatile species such as $\mathrm{CH}_{3} \mathrm{OH}$ (Garay et al. 2000; Jørgensen et al. 2004). The dissociation of $\mathrm{SiO}$ by shocks would require velocities $\gtrsim 50-60 \mathrm{~km} \mathrm{~s}^{-1}$ (i.e., J shocks), however.

If we consider both processes, i.e., the injection of grain material and the disruption of molecules in gas phase, we can conclude that an increase in the typical velocity regime of shocks $\left(v_{\text {shock }}\right)$ will certainly favour an enhancement of the abundance of $\mathrm{SiO}$ in the shocked gas $\left(\left.X(\mathrm{SiO})\right|_{\text {shock }}\right)$. This will be at the expense of increasing the SiO-to- $\mathrm{CH}_{3} \mathrm{OH}$ abundance ratio in molecular gas. Therefore, a variation in the SiO-to$\mathrm{CH}_{3} \mathrm{OH}$ intensity ratio can be taken as evidence for a change in $v_{\text {shock}}$. A quantitative comparison of the $\mathrm{SiO}$ and $\mathrm{CH}_{3} \mathrm{OH}$ maps of IC 342 could thus help to discern if the typical shock velocity regime changes across the galaxy disk.

\subsection{Tracers of shocks in IC 342: $\mathrm{SiO}$ and $\mathrm{CH}_{3} \mathrm{OH}$}

Meier \& Turner (2005) used the OVRO interferometer to map the emission of the $2_{\mathrm{k}}-1_{\mathrm{k}}$ line of $\mathrm{CH}_{3} \mathrm{OH}$ in IC 342 with a resolution similar to that of the $\mathrm{SiO}$ map. This spatial resolution is comparable to the typical GMC-scales ( $\sim 80 \mathrm{pc})$. We have compared the emission of $\mathrm{SiO}(2-1)$ to that of $\mathrm{CH}_{3} \mathrm{OH}\left(2_{\mathrm{k}}-1_{\mathrm{k}}\right)$ in order to derive the SiO-to- $\mathrm{CH}_{3} \mathrm{OH}$ intensity ratio in GMCs A-to-E. Figure 8 represents these ratios as 

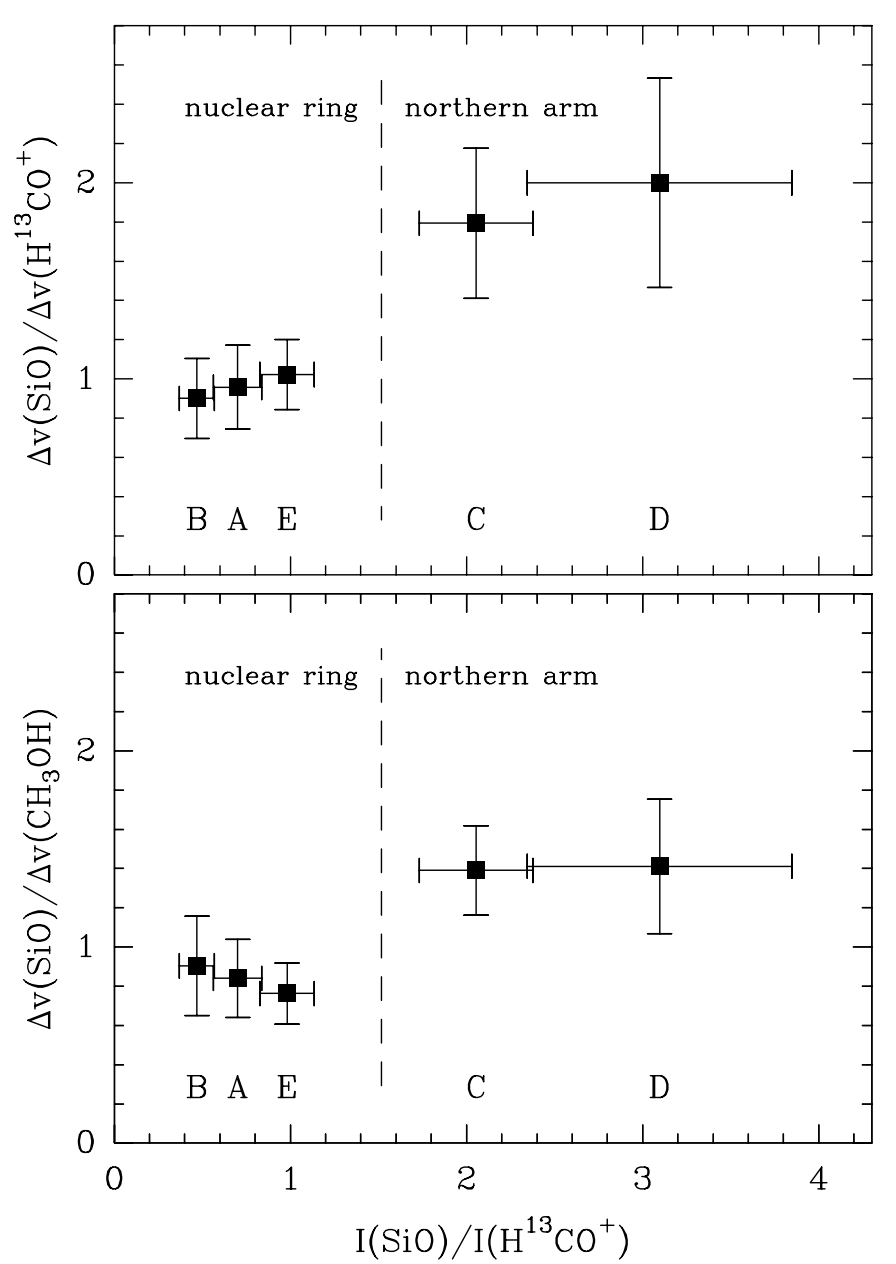

Fig. 9. $\mathrm{SiO}$-to- $\mathrm{H}^{13} \mathrm{CO}^{+}$and $\mathrm{SiO}-$ to- $\mathrm{CH}_{3} \mathrm{OH}$ velocity width ratios measured for GMCs A-to-E. These ratios are represented as a function of the $\mathrm{SiO}-$ to- $\mathrm{H}^{13} \mathrm{CO}^{+}$integrated intensity ratio, $R_{I}$.

a function of $R_{I}$ for the nuclear ring and northern spiral arm GMCs. As is shown in Fig. 8, the reported nearly order of magnitude change in $R_{I}$ is not corresponded with a similar change in the $I(\mathrm{SiO}) / I\left(\mathrm{CH}_{3} \mathrm{OH}\right)$ ratio between the northern spiral arm and the nuclear ring. The $I(\mathrm{SiO}) / I\left(\mathrm{CH}_{3} \mathrm{OH}\right)$ ratio is fairly constant and close to $\sim 0.14^{1}$ for all GMCs, though we find tentative evidence for a larger value in GMC D $(\sim 0.24 \pm 0.07)$. Taken together these results indicate that, at first order, the N-S gradient measured in $R_{I}$ between the northern spiral arm and the nuclear ring can be mostly attributed to a variation of $f_{\text {shock }}$.

The line ratios above are derived from velocity-integrated intensities and are beam-averaged on scales that are typical of GMC-like units at the distance of IC 342. However, the analysis of the line profiles of $\mathrm{SiO}, \mathrm{CH}_{3} \mathrm{OH}$ and $\mathrm{H}^{13} \mathrm{CO}^{+}$can provide information on the shock velocity regime on scales smaller than the beam. Figure 9 displays the $\mathrm{SiO}-$ to- $\mathrm{H}^{13} \mathrm{CO}^{+}$and SiO-to$\mathrm{CH}_{3} \mathrm{OH}$ velocity-width ratios derived for GMCs A-to-E. These ratios show a different behaviour in the nuclear ring and in the

1 Assuming the $\mathrm{CH}_{3} \mathrm{OH}$ column densities given by Meier et al. (2005) these intensity ratios translate into $\mathrm{SiO}-\mathrm{CH}_{3} \mathrm{OH}$ column density ratios of $\sim 0.01-0.04$ for $n \gtrsim 5 \times 10^{4} \mathrm{~cm}^{-3}$ ( $\sim 3$ times larger if $n \sim 10^{4} \mathrm{~cm}^{-3}$ ). northern spiral arm. In the ring, linewidths and velocity centroids for $\mathrm{SiO}, \mathrm{H}^{13} \mathrm{CO}^{+}$and $\mathrm{CH}_{3} \mathrm{OH}$ are virtually identical. In contrast, $\mathrm{SiO}$ lines are a factor of $\sim 2$ wider than those of $\mathrm{H}^{13} \mathrm{CO}^{+}$in the northern spiral arm, as reported in Sect. 3.2.2. $\mathrm{CH}_{3} \mathrm{OH}$ lines represent a case intermediate between these two extremes: $\mathrm{SiO}$ lines are a factor of $\sim 1.4$ wider than those of $\mathrm{CH}_{3} \mathrm{OH}$ in the spiral arm. The differences between $\mathrm{SiO}$, $\mathrm{CH}_{3} \mathrm{OH}$ and $\mathrm{H}^{13} \mathrm{CO}^{+}$are evident in the linewidths, but velocity centroids are the same within the errors. As discussed in Sect. 7, this suggests that the apparent turbulence of shocked molecular gas is enhanced compared to the more quiescent gas.

While it seems that the enhancement of $f_{\text {shock }}$ in the northern spiral arm explains the bulk of the reported increase of $X(\mathrm{SiO})$ in this region, the observed differences in the line profiles of $\mathrm{SiO}$ and $\mathrm{CH}_{3} \mathrm{OH}$ suggest that a fraction of the shocked gas in the arm presents a higher $\left.X(\mathrm{SiO})\right|_{\text {shock }}$. This implies that $v_{\text {shock }}$ would be a factor of $\sim 2$ larger in the spiral arm region.

\section{SiO emission in galaxies}

There is ample observational evidence that $\mathrm{SiO}$ thermal emission can be locally enhanced in star forming molecular clouds of our Galaxy (Martín-Pintado et al. 1992; Bachiller et al. 2001). More recently, $\mathrm{SiO}$ has been revealed to be a tracer of shock chemistry also in galaxy nuclei, including our own Galaxy (Sage \& Ziurys 1995; Martín-Pintado et al. 1997; García-Burillo et al. 2000, 2001b; Usero et al. 2004). We revise below the various driving mechanisms which have been proposed to explain the onset of shock chemistry in the Galaxy and in galaxies in general, and study their applicability to the nucleus of IC 342 .

\subsection{SiO emission in the disk of our Galaxy}

$\mathrm{SiO}$ thermal emission is observed towards star-forming clouds in the disk of our Galaxy. In particular, the strongest $\mathrm{SiO}$ emitters are the bipolar outflows located around protostellar objects (Martín-Pintado et al. 1992; Bachiller et al. 2001). In the first stages of the star formation process, bipolar outflows interact with the ambient molecular gas, inducing molecular shocks which are able to increase the abundances of some molecular species (like $\mathrm{SiO}$ and $\mathrm{CH}_{3} \mathrm{OH}$ ) by several orders of magnitude with respect to quiescent gas (Bachiller et al. 1995, 1997).

The abundance of $\mathrm{SiO}$ in IC 342 reaches the largest value in the northern spiral arm (Sect. 4), where the evidence of ongoing star formation is scarce (Sect. 3.1). However, it could be argued that $\mathrm{SiO}$ emission in IC 342 is probing the deeply embedded phase of a young star formation episode. The episode would be spread on scales of $\sim$ a few 100 pc and would not be visible in $\mathrm{H} \alpha$ or thermal radio-continuum. Although with these tight constraints this explanation is unlikely, we can discard it on more quantitative grounds comparing the $\mathrm{SiO}$ emission in IC 342 with a subset of bipolar outflows of the Galaxy (Table 2). For the reasons explained below, we have purposely selected outflows for which there are maps of both $\mathrm{SiO}(2-1)$ and $\mathrm{CH}_{3} \mathrm{OH}\left(2_{\mathrm{k}}-1_{\mathrm{k}}\right)$ available in the literature. Furthermore, since $\mathrm{SiO}$ luminosities are larger for more massive and younger objects, the outflows have been chosen to cover a wide range 
Table 2. $\mathrm{SiO}(2-1)$ and $\mathrm{CH}_{3} \mathrm{OH}\left(2_{\mathrm{k}}-1_{\mathrm{k}}\right)$ luminosities of a sample of bipolar outflows. Column 2: mass of the class 0 object; Col. 3: luminosity of the class 0 object; $\mathrm{Col} .4$ : $\mathrm{SiO}$ luminosity integrated within the $\mathrm{SiO}$ emitting region of the outflow; Col. 5: $\mathrm{SiO}$-to- $\mathrm{CH}_{3} \mathrm{OH}$ total luminosity ratio; Col. 6: surface density of outflows required to obtain the mean $I(\mathrm{SiO})$ in the northern spiral arm+nuclear ring of IC 342; Col. 7: references: $a$ : Bachiller et al. (2001); $b$ : Gueth et al. (1997); $c$ : Fuente et al. (2005b); $d$ : Codella \& Bachiller et al. (1999).

\begin{tabular}{|c|c|c|c|c|c|c|}
\hline (1) & (2) & (3) & (4) & (5) & (6) & (7) \\
\hline Source & $\begin{array}{c}M_{\star} \\
\left(M_{\odot}\right)\end{array}$ & $\begin{array}{l}L_{\star} \\
\left(L_{\odot}\right)\end{array}$ & $\begin{array}{c}L_{\mathrm{SiO}} \\
\left(\mathrm{K} \mathrm{km} \mathrm{s}^{-1} \mathrm{pc}^{2}\right)\end{array}$ & $L_{\mathrm{SiO}} / L_{\mathrm{CH}_{3} \mathrm{OH}}$ & $\begin{array}{c}N_{\text {outf }} \\
\text { (outflows } \mathrm{pc}^{-2} \text { ) }\end{array}$ & Ref. \\
\hline L 1157 (blue lobe) & 0.2 & 11 & 0.19 & 0.38 & 4.9 & $a, b$ \\
\hline NGC 7129-FIRS2 & 5 & 500 & & & & $c$ \\
\hline outflow 1 & & & 0.21 & 0.14 & 4.4 & \\
\hline outflow 2 & & & 0.08 & 0.10 & 11.6 & \\
\hline CB3 & 4 & 930 & 2.37 & 0.45 & 0.4 & $d$ \\
\hline
\end{tabular}

in mass and age of the protostars. It is assumed that the largest $\mathrm{SiO}$-to- $\mathrm{CH}_{3} \mathrm{OH}$ luminosity ratios correspond to the less evolved objects (Bergin et al. 1998).

The mean SiO intensity of the northern spiral arm and the nuclear ring of IC 342, derived inside the region defined by the lowest contour of Fig. 2, is $\sim 0.93 \mathrm{~K} \mathrm{~km} \mathrm{~s}^{-1}$. We have then inferred the surface density of outflows which would be required to reproduce the observed $\mathrm{SiO}$ intensity in IC 342. As is shown in Table 2, this number density of outflows range from $\sim 0.4$ to $\sim 12$ outflows $\mathrm{pc}^{-2}$. Though in star-forming regions of our Galaxy like OMC2/3 or NGC 2068, surface densities of a few outflows $\mathrm{pc}^{-2}$ have been reported on scales of 1-2 pc $^{2}$ (Reipurth et al. 1999; Mitchell et al. 2001), it is very unlikely that these average surface densities can be attained in the $\mathrm{SiO}$ disk of IC 342 , which is several hundred $\mathrm{pc}^{2}$ in extent.

Furthermore the star forming rate (SFR) of IC 342 poses tight constraints on the upper limit to the expected density of outflows. The SFR in a galaxy can be easily estimated from the FIR luminosity (Kennicutt 1998). In the case of IC 342, $L_{\mathrm{FIR}}=$ $1.5 \times 10^{9} L_{\odot}$ in the central $r \sim 15^{\prime \prime}$ (Becklin et al. 1980; scaled to $D=3.3 \mathrm{Mpc})$. Assuming that the northern spiral arm and nuclear ring are the main contributors to the SFR of IC 342, we estimate for this region a SFR density of $3 \times 10^{-6} M_{\odot} \mathrm{yr}^{-1} \mathrm{pc}^{-2}$. From the SFR density above, we can then derive an upper limit to the surface density of outflows, assuming a Salpeter law for the $\operatorname{IMF}\left(\mathrm{d} \log (N) / \mathrm{d} \log (m)=-2.35\right.$ over $\left.m=0.1-100 M_{\odot}\right)$ and a timescale for the pre-stellar phase of $\sim 10^{4} \mathrm{yr}$. The derived upper limit to the total density of outflows provided by the SFR in IC 342 is $\sim 9 \times 10^{-2}$ outflows $\mathrm{pc}^{-2}$. Even for outflows like CB3 (or any other $\mathrm{SiO}$ luminous YSO), the required density would be $\sim 5$ times larger than that provided by the SFR of IC 342. Moreover, massive young bipolar outflows like CB3 should be the minority among YSOs: for a standard Salpeter IMF, only $4 \%$ of the objects would have masses above $1 M_{\odot}$. In addition, the observed $\mathrm{SiO}$-to- $\mathrm{CH}_{3} \mathrm{OH}$ average ratio of IC 342 $(\sim 0.14$; see Fig. 8$)$ is similar to YSOs which are much more evolved than CB3.

In summary, we can discard the interpretation of the largescale shocks in IC 342 in terms of a collection of YSOs associated with an embedded star formation episode in the inner $r \sim 320$ pc disk of the galaxy.

\subsection{SiO emission in galactic nuclei}

\subsubsection{The center of our Galaxy}

The nucleus of our Galaxy shows widespread SiO emission that, in contrast to that observed in the Galactic Disk, is not related to recent star formation. The first large-scale $\mathrm{SiO}$ observations of the Galactic Center (GC) of Martín-Pintado et al. (1997) detected the emission of the $\mathrm{SiO}(1-0)$ line in a $\sim 150$ pc-diameter circumnuclear disk. In a later paper, Hüttemeister et al. (1998) detected the $\mathrm{SiO}(2-1)$ emission in 32 GC molecular clouds located inside a 300 pc-diameter disk which extends from Sgr C to a position at slightly higher positive longitudes than Sgr B2. High $\mathrm{SiO}$ abundances are derived for these clouds ( $\sim$ several $10^{-10}$-several $\left.10^{-9}\right)$, indicative of shock chemistry. This scenario has received further support from recent observations revealing the complex alcohol chemistry of GC clouds (Martín-Pintado et al. 2001; Requena-Torres et al. 2005, in prep.). In these clouds the large abundances of ethanol $\left(\mathrm{C}_{2} \mathrm{H}_{5} \mathrm{OH}\right)$ and $\mathrm{CH}_{3} \mathrm{OH}$ evidence the erosion of dust grain mantles on large scales.

Besides the long reported similarities between our Galaxy and IC 342 (e.g., Downes et al. 1992), the inner few hundred $\mathrm{pc}$ in the two galaxies seem to be the scenario of large-scale molecular shocks leading to dust grain processing. Furthermore, the efficiency of shocks appear to be comparable in both galaxies. Despite the different spatial resolution of $\mathrm{SiO}$ observations in IC $342(\sim 80 \mathrm{pc})$ and in our Galaxy $(\sim 2 \mathrm{pc})$, the derived $\mathrm{SiO}$ abundances are similar in the two objects. Moreover, the SiO-to- $\mathrm{CH}_{3} \mathrm{OH}$ abundance ratios are also similar in the GC clouds $(\sim 0.01-0.03)^{2}$ and in IC $342(\sim 0.01-0.04$; see Sect. 5.2).

These similarities taken together, we can hypothesize that the mechanism explaining the onset of large-scale molecular shocks in the GC and in IC 342 is likely to be the same. Shocks identified in GC clouds, also unrelated to ongoing star formation, have been attributed different causes, however: the interaction with GC non-thermal filaments, with supernovae remnants or with the expansion bubbles created by

\footnotetext{
${ }^{2}$ Calculated from $\mathrm{SiO}$ and $\mathrm{C}_{2} \mathrm{H}_{5} \mathrm{OH}$ column densities measured in GC clouds (Martín-Pintado et al. 1997, 2001), assuming a constant $\mathrm{C}_{2} \mathrm{H}_{5} \mathrm{OH}$-to- $\mathrm{CH}_{3} \mathrm{OH}$ abundance ratio of $\sim 0.05$ (Requena-Torres et al. 2005 , in prep.).
} 
Table 3. $\mathrm{SiO}$ emission in external galaxies. Column 2: $\mathrm{SiO}$ abundance averaged within $28^{\prime \prime}$ (from observations with the IRAM $30 \mathrm{~m}$ telescope); the averaging scale is $\sim 0.5 \mathrm{kpc}$, for NGC 253, IC 342 and M 82, and $\sim 2 \mathrm{kpc}$ for NGC 1068; Col. 3: components resolved with the PdBI contributing to the global $\mathrm{SiO}$ emission; Col. 4: $\mathrm{SiO}$ abundance in the resolved components; Col. 5: mechanism proposed to be enhancing the $\mathrm{SiO}$ abundance; underlying physical process, in brackets (DW stands for "density waves"); Col. 6: references: $a$ : García-Burillo et al. (2000); $b$ : this work; $c$ : García-Burillo et al. (2001b); $d$ : Usero et al. (2004); $e$ : Martín-Pintado et al. (2005, in prep.).

\begin{tabular}{|c|c|c|c|c|c|}
\hline (1) & (2) & (3) & (4) & (5) & (6) \\
\hline Galaxy & $\begin{array}{c}\langle X(\mathrm{SiO})\rangle_{30 \mathrm{~m}} \\
\left(10^{-10}\right)\end{array}$ & Resolved components & $\begin{array}{l}X(\mathrm{SiO}) \\
\left(10^{-10}\right)\end{array}$ & SiO Chemistry & Ref. \\
\hline \multirow[t]{3}{*}{ NGC 253} & $1-3$ & & & & $a, e$ \\
\hline & & $r \sim 60 \mathrm{pc}$ inner ring & $1-2$ & shocks [YSOs / DW] & \\
\hline & & $r \sim 300 \mathrm{pc}$ outer ring & $5-15$ & shocks [DW] & \\
\hline \multirow[t]{3}{*}{ IC 342} & $3-7$ & & & & $b, e$ \\
\hline & & $r \sim 50 \mathrm{pc}$ ring & $2-7$ & shocks [DW] & \\
\hline & & $150 \mathrm{pc}$ length arm & $10-40$ & shocks [DW] & \\
\hline \multirow[t]{3}{*}{ M 82} & 0.5 & & & & $c, e$ \\
\hline & & $r \sim 75$ pc shell & $0.4-1$ & shocks [galactic outflow] & \\
\hline & & $500 \mathrm{pc}$ length chimney & $2-3.5$ & shocks [galactic outflow] & \\
\hline \multirow[t]{3}{*}{ NGC 1068} & 7 & & & & $d, e$ \\
\hline & & $r \sim 1-1.5 \mathrm{kpc}$ ring & $\sim$ a few & shocks [YSOs / DW] & \\
\hline & & $r \sim 200 \mathrm{pc}$ disk & $\sim 50$ & $\mathrm{XDR}[\mathrm{AGN}] /$ shocks [DW] & \\
\hline
\end{tabular}

Wolf-Rayet stars have been discussed by Martín-Pintado et al. (1997). Hüttemeister et al. (1998), using observations that extended over a wider region in the $\mathrm{GC}$, found the largest $\mathrm{SiO}$ abundances where the likelihood of cloud-cloud collisions, induced by the Galactic bar potential, is the highest. As discussed in Sects. 6.2.2 and 7, the latter scenario is the preferred one in IC 342.

\subsubsection{The nuclei of external galaxies}

Mauersberger \& Henkel (1991) detected the emission of $\mathrm{SiO}(2-1)$ in the starburst galaxy NGC 253, using the $30 \mathrm{~m}$ IRAM telescope with a resolution of $\sim 28^{\prime \prime}$. This detection, the first reported for $\mathrm{SiO}$ outside the Milky Way, was followed by a 9-galaxy survey made with the NRAO $12 \mathrm{~m}$ antenna by Sage $\&$ Ziurys (1995) with $\sim 67 "$ spatial resolution (equivalent to $\sim 0.5-2 \mathrm{kpc})$. In this survey, where 5 galaxies were detected in $\mathrm{SiO}$, Sage \& Ziurys (1995) found no correlation between the abundance of $\mathrm{SiO}$, characterized in their work by the $\mathrm{SiO} / \mathrm{N}_{2} \mathrm{H}^{+}$ ratio, and the efficiency of star formation (given by the SFR per unit dynamical mass, i.e., SFR/ $M_{\text {dyn }}$ ). This surprising result was at odds with the classical framework where shock chemistry is driven by YSOs in star forming regions of the Galactic disk. The global SiO abundance measured on 0.5-2 kpc-scales is seen to vary significantly among starburst galaxies: it can reach $\sim 10^{-9}$ in IC 342 , whereas it is $1 / 20$ of this value in M 82 (see Table 3 and Martín-Pintado et al. 2005, in prep.).

The occurrence of large-scale molecular shocks may arise at different stages during the typical lifetime of a starburst episode (Rieke et al. 1988; García-Burillo \& Martín-Pintado 2001a). In the pre-starburst phase (I), density wave instabilities induce gravitational torques and drive the infall of large amounts of gas towards the nucleus. Large-scale shocks may be at work related to an enhanced compression of gas and an increased rate of cloud-cloud collisions in the potential wells of spiral arms and/or bars. Once the first massive stars are formed in a second phase (II), bipolar outflows can produce locally molecular shocks in YSOs. In a later stage, corresponding to an evolved starburst, the elevated rate of SN explosions may lead to the disruption of the disk during the expansion of the so-called hot bubble. Episodes of mass injection from the disk into the halo could be accompanied by molecular shocks.

The advent of high-resolution $\mathrm{SiO}$ images has been key to help discern the different sources of shock chemistry in external galaxies and thus identify an evolutionary path along the starburst sequence depicted above (see Table 3 and references therein). In particular, the puzzling result issued from first single-dish $\mathrm{SiO}$ surveys starts to be understood when observations allow us to zoom in on molecular galaxy disks on scales $\lesssim 100$ pc:

- Large-scale shocks near the outer Inner Lindbland Resonance (oILR) of the NGC 253 stellar bar can account for the outer $\mathrm{SiO}$ disk of this galaxy, which extends well beyond the nuclear starburst. The outer disk SiO emission gives away the pre-starburst phase (I) in NGC 253 (García-Burillo et al. 2000). More recently, the detection of $\mathrm{SO}_{2}$, NS and $\mathrm{NO}$ emission and the analysis of the sulfur chemistry in NGC 253 have confirmed that shocks are at work in the nucleus of this galaxy (Martín et al. 2003, 2005).

- Outflows driven by YSOs during phase II can partly explain the measured average abundances of $\mathrm{SiO}$ in the inner disk of NGC 253 (García-Burillo et al. 2000). However, the bulk of the $\mathrm{SiO}$ emission likely stems from molecular cloud shocks in the inner Inner Lindbland Resonance (iILR) of the NGC 253 stellar bar.

- The emission of SiO extends noticeably out of the galaxy

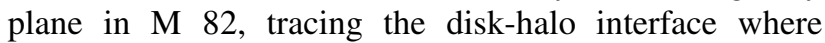
episodes of mass injection from the disk are building up 
the gaseous halo (phase III) (García-Burillo et al. 2001b). The PdBI maps of M 82 made in $\mathrm{SiO}$ and $\mathrm{HCO}$ illustrate how two different gas chemistry scenarios can be simultaneously at play in the same galaxy though at different locations: shocks in the disk-halo interface and PDR chemistry in the galaxy disk which hosts an evolved starburst (García-Burillo et al. 2002; Fuente et al. 2005a).

The case of the Seyfert 2 galaxy NGC 1068 has been studied by Usero et al. (2004), who estimate a large abundance of $\mathrm{SiO}$ in the $r \sim 200 \mathrm{pc} \mathrm{CND}$ of the galaxy $\left(X(\mathrm{SiO}) \sim\right.$ a few $\left.10^{-9}\right)$. The enhancement of $\mathrm{SiO}$ is attributed by Usero et al. (2004) to the evaporation of very small (10 ̊) silicate grains (VSG) by $\mathrm{X}$-rays. Alternatively, silicon chemistry could be also driven by pre-starburst shocks related with the density wave-resonances of the CND (García-Burillo et al. 2005, in prep.).

As extensively argued in Sect. 6.2.1, the $\mathrm{SiO}$ emission in IC 342 cannot be explained by ongoing star forming activity (phase II). The M 82 scenario (phase III) can be also ruled out in IC 342. SiO emission in the disk of IC 342 extends well beyond the distribution of supernovae remnants (SNRs) (Condon et al. 1982; Bregman et al. 1993). Furthermore, the low supernovae rate of IC $342\left(<0.04 \mathrm{yr}^{-1}\right.$ for $D=3.3 \mathrm{Mpc}$; Condon et al. 1982) yields a $\sim 70$ times smaller energy deposition by the SN of IC 342 compared to M 82. Finally, X-rays are not expected to be a dominating agent in the chemistry of molecular gas in IC 342 either. Nearly 35\% of the observed hard X-ray emission in IC $342(2 \mathrm{keV} \leq E \leq 10 \mathrm{keV})$ comes from a circumnuclear disk of $r \sim 8^{\prime \prime}$ (Bauer et al. 2003). The hard X-ray luminosity of this source is three orders of magnitude lower than that of NGC 1068 (Ogle et al. 2003), however. Furthermore, the $6.4 \mathrm{keV} \mathrm{Fe} \mathrm{K} \alpha$ line, which probes the processing of neutral gas by X-rays, is absent from the spectrum of IC 342 .

The exclusion of all the alternative explanations leads us to conclude that the large-scale shocks identified in the inner $r \sim 320$ pc of IC 342 arise in the pre-starburst phase (I) (see Sect. 8). The following section discusses the efficiency of density waves in producing shocks in molecular gas in IC 342.

\section{Density waves and shocks in IC 342}

The IC 342 bar shapes the distribution and kinematics of molecular gas in the central $r \sim 320 \mathrm{pc}$ of the galaxy (Turner \& Hurt 1992; Schinnerer et al. 2003; Meier \& Turner 2005; this work). The spiral-like morphology of the molecular disk and the detection of non-circular motions $\left(\gtrsim 50-60 \mathrm{~km} \mathrm{~s}^{-1}\right.$ in the northern spiral arm, deprojected onto the galaxy plane) are reminiscent of the typical bar-driven dynamics. The $\mathrm{SiO}$ abundances measured in the inner molecular disk of IC 342 proves unambiguously that the bar is producing large-scale molecular shocks. However, the detection of $\mathrm{SiO}$ emission constrains the velocity regime of shocks to lie between $\gtrsim 15-20 \mathrm{~km} \mathrm{~s}^{-1}$ (for grain cores to be significantly disrupted) and $\lesssim 50-60 \mathrm{~km} \mathrm{~s}^{-1}$ (to prevent dissociation of $\mathrm{SiO}$ molecules), i.e., a velocity range which lies significantly below the lower limit set to the noncircular motions measured across the IC 342 bar. Furthermore, the detection of $\mathrm{CH}_{3} \mathrm{OH}$ emission across the bar suggests that the shocked molecular gas emitting in $\mathrm{CH}_{3} \mathrm{OH}$ cannot be characterized by $v_{\text {shock }} \gtrsim 50-60 \mathrm{~km} \mathrm{~s}^{-1}$. These observational constraints imply that the input kinetic energy provided by streaming motions must be first dissipated making room for a lower velocity regime that corresponds to the emission of the molecular shock tracers observed across the IC 342 bar.

Large-scale shocks driven by density waves have been long predicted by numerical simulations of spiral/barred galaxies, made following either hydrodynamical schemes (Roberts 1969; Athanassoula 1992) or ballistic ones (Casoli \& Combes 1982; Combes \& Gerin 1985). In ballistic models, which likely provide a more realistic representation of the clumpy dense ISM in galaxies, molecular shocks should arise subsequently after cloud-cloud collisions. The number of collision events is enhanced by an increase of orbit crowding along the potential well of the bar. As noted in Sects. 3.2.2 and 5.2, $\mathrm{SiO}, \mathrm{H}^{13} \mathrm{CO}^{+}$ and $\mathrm{CH}_{3} \mathrm{OH}$ lines have all similar velocity centroids, but different linewidths over the spiral arm region. The largest widths correspond to $\mathrm{SiO}$ (whose lines are a factor of 2 larger than those of $\mathrm{H}^{13} \mathrm{CO}^{+}$), with $\mathrm{CH}_{3} \mathrm{OH}$ representing an intermediate case. This suggests that the apparent turbulence of shocked molecular gas, traced by $\mathrm{SiO}$ and $\mathrm{CH}_{3} \mathrm{OH}$, is enhanced compared to the state of the more quiescent dense gas medium (traced by $\mathrm{H}^{13} \mathrm{CO}^{+}$). This effect, especially relevant in the northern spiral arm region (see Fig. 5), suggests that the molecular shocks arise at a stage of turbulent dissipation and not during the early phase of the encounter, when cloud-cloud relative velocities may lead to the dissociation of $\mathrm{SiO}$ and $\mathrm{CH}_{3} \mathrm{OH}$.

Assuming that the internal structure of the colliding molecular clouds is highly clumpy (e.g., Falgarone \& Puget 1985), it is plausible to assume that a fraction of the kinetic energy dissipated during a cloud-cloud collision can cascade down to smaller scales. This would increase the turbulent motions of clumps composing the end product of any cloud-cloud collision. For the simulations, Kimura \& Tosa (1996) have indeed found indications that the internal turbulence of clumpy colliding molecular clouds can increase after an encounter (see also Bonnell et al. 2005). The input kinetic energy typically involved in a cloud-cloud collision should be much larger in the northern spiral arm than in the nuclear ring. Once dissipated, these different input energies would end up producing a higher turbulence in the shocked molecular gas of the spiral arm $\left(\sigma_{v} \sim 20-25 \mathrm{~km} \mathrm{~s}^{-1}\right.$; see Sect. 3.2.2) compared to the nuclear ring $\left(\sigma_{v} \sim 10 \mathrm{~km} \mathrm{~s}^{-1}\right.$; see Sect. 3.2.2). As observed, in this scenario the largest difference between these two regions would be the amount of shocked molecular gas mass $\left(f_{\text {shock }}^{\text {arm }} \sim 5-7 \times f_{\text {shock }}^{\text {ring }}\right.$; see Sect. 5.2) and not the shock velocity regime, which is here equal to $\sigma_{v}\left(\sigma_{v}^{\text {arm }} \sim 2 \times \sigma_{v}^{\text {ring }}\right.$; see Sect. 5.2).

The fraction of molecular gas actually involved in the prestarburst shocks produced by the IC 342 bar defines the relevance of this process. We can estimate this fraction in IC 342 making use of Eq. (1). Given the similar properties of shocks in the GC and IC 342 (Sect. 6.2.1), we can reasonably adopt a value of $\left.X(\mathrm{SiO})\right|_{\text {shock }}$ in IC 342 similar to that estimated in the GC clouds on $\sim 1-2$ pc-scales by Hüttemeister et al. (1998): $\left.X(\mathrm{SiO})\right|_{\text {shock }} \gtrsim 10^{-8}$. From the values derived for $X(\mathrm{SiO})$ in 
IC 342 on $\sim 80$ pc scales, we conclude that $f_{\text {shock }} \lesssim 0.1$ in the northern spiral arm and $\lesssim 0.02$ in the nuclear ring. In Appendix A, we estimate the rate of energy dissipated by shocks in the gas over the spiral arm region of this galaxy. While this estimate is rather approximate, the resulting picture underlines the potential role of large-scale shocks in draining energy from the gas inflowing towards galactic nuclei.

\section{Conclusions and perspectives}

The high-resolution images showing the emission of $\mathrm{SiO}$ in the inner $r \sim 320$ pc disk of IC 342 reveal the onset of large-scale molecular shocks driven by the bar potential of this galaxy. The variation of the estimated $\mathrm{SiO}$ abundance inside the mapped region (from $\sim 10^{-10}$ to $\sim 10^{-9}$ ) and the comparison with other molecular tracers $\left(\mathrm{CH}_{3} \mathrm{OH}\right.$ and $\left.\mathrm{H}^{13} \mathrm{CO}^{+}\right)$indicate that shocks process with uneven efficiency (see Sects. 4 and 5) the molecular gas reservoir of IC 342 . The shocks seem to arise during cloud-cloud collisions at the stage when kinetic energy has partly dissipated in turbulent motions. The mass of molecular gas locally involved in shocks over the spiral arm region of IC 342 could amount to $10 \%$ of the dense gas reservoir. Taken together, these results underline the relevant role that largescale molecular shocks can play in shaping the evolution of gas disks.

These observations illustrate the occurrence of molecular shocks in galaxies that are not related with ongoing star formation. SiO emission in IC 342 traces a pre-starburst phase in molecular gas. Pre-starburst shocks have also been identified to be responsible for the intense ro-vibrational $\mathrm{H}_{2}$ lines detected at $2 \mu \mathrm{m}$ in 2 prototypical mergers: Arp 220 and NGC 6240 (Rieke et al. 1985). More recently, Haas et al. (2005) have reported the detection of widespread emission of the $v=0-0$ $\mathrm{S}(3)$ line of $\mathrm{H}_{2}$ at $9.66 \mu \mathrm{m}$ in the overlap region of the Antennae galaxy pair. Haas et al. (2005) interpret this emission as a tracer of shocks that will give rise to the first generation of stars in the region. In the case of the Antennae, Haas et al. (2005) hypothesize that shocks may not be the result of direct collisions of molecular clouds but arise instead from an overpressured medium that remains of the collisions between H I clouds (Jog \& Solomon 1992).

In contrast to the Antennae, the bulk of the shocked gas in IC 342 is not expected to produce on-site star formation due to the inhibiting action of strong shear over the spiral arms. $\mathrm{SiO}$ emission in IC 342 traces the sites where molecular clouds dissipate a fraction of their energy through collisions; the energy loss helps the gas to fall to the nuclear ring where it will feed the starburst. The $\mathrm{SiO}$ map of IC 342 provides a snapshot view of the pre-starburst phase during the fueling process driven by density waves. Higher-resolution observations are required to provide new constraints on the details of how density waves operate to produce molecular shocks. In particular, we expect that the efficiency of shocks changes transversally to the spiral arms, as cloud orbits are re-oriented by the bar potential. Because of its closeness, favourable orientation and well defined spiral pattern, IC 342 is a good target for follow-up studies.

High-resolution $\mathrm{SiO}$ imaging is key to discern the different sources of shock chemistry which are activated at different locations and at different moments in galaxy disks during a starburst event. Being more than a mere tracer of $e x$ otic chemistry, $\mathrm{SiO}$ allows to probe unambiguously the regions where dust grains are being destroyed in galaxies due to the action of density waves, star formation, galactic outflows or Xrays (García-Burillo et al. 2000, 2001b; Usero et al. 2004). The study of the feedback influence of these phenomena in nearby galactic disks is paramount to constrain models of evolution and formation of galaxies at higher redshifts.

Acknowledgements. We acknowledge the IRAM staff for help provided during the observations and for data reduction. This paper has been partially funded by the Spanish MCyT under projects DGES/AYA2000-0927, ESP2001-4519-PE, ESP2002-01693, PB1998-0684, AYA2002-01241, ESP2002-01627 and AYA200210113E. This research has made use of NASA's Astrophysics Data System and NASA/IPAC Extragalactic Database (NED).

\section{Appendix A: Energy dissipation by large-scale molecular shocks in IC 342}

Stellar bars can remove energy and angular momentum from gas through gravitational torques and shocks. Gravity torques from the stellar potential on the gas are expected to be the most relevant drivers of gas inflow in galaxies. Torques created by large-scale stellar bars alone or helped by other secondary mechanisms, such as nested stellar bars, dynamical friction or viscosity, can drive gas inflow and feed the central $a c$ tivity of the inner $1 \mathrm{kpc}$ playground in galactic nuclei (e.g., García-Burillo et al. 2005). Large-scale shocks may also contribute significantly to the loss of energy. In this appendix, we make a rough estimate of the rate of energy loss produced locally by the onset of large-scale shocks in the gas over the spiral arm region of IC 342 .

A shock-front propagating in a gas medium dissipates ordered kinetic energy into heat. For large Mach numbers ( $v_{\text {shock }} \gg$ sound speed), the kinetic energy lost per unit shocked mass is $\sim v_{\text {shock }}^{2} / 2$, both in radiative and non-radiative shocks. (e.g. Draine \& McKee 1993). The rate of energy dissipated per unit total mass due to shocks would be:

$\dot{e}_{\mathrm{s}}=-\frac{f}{\tau_{\mathrm{SiO}}} \frac{v_{\text {shock }}^{2}}{2}$

$f$ is the total fraction of shocked gas (including the dense molecular component traced by $\mathrm{SiO}$ and the more diffuse gas), while $\tau_{\mathrm{SiO}}$ is the depletion timescale of $\mathrm{SiO}$ onto grains $\left(\sim 10^{4} \mathrm{yr}\right.$; Bergin et al. 1998). Since gas-phase $\mathrm{SiO}$ depletes quickly onto grains, the shocked gas traced by $\mathrm{SiO}$ must have been shocked within a time $\tau_{\mathrm{SiO}}$. The expected values of $f$ range between $f_{\text {shock }}$ (if dense and diffuse gas are assumed to be shocked to the same extent) and $\sim 0.1 \times f_{\text {shock }}$ (if we assume that only the dense gas is preferentially shocked; in this case we take a fraction of dense gas of $\sim 10 \%$ in IC 342 from Schulz et al. 2001). Assuming a typical $v_{\text {shock }}$ of $25 \mathrm{~km} \mathrm{~s}^{-1}$, $\dot{e}_{\mathrm{s}}=-(0.3-3) \times 10^{3} \mathrm{~km}^{2} \mathrm{~s}^{-2} \mathrm{Myr}^{-1}$ for a corresponding range in $f=0.01-0.1$.

Assuming quasi-circular motion, we can estimate a dissipation timescale, $\tau_{\mathrm{s}}$, for the shocks to drain the specific energy 
of the gas, $e\left(e \simeq v_{\varphi}^{2} / 2+\phi\right.$, where $v_{\varphi}$ is the azimuthal velocity and $\phi(r)$ is the mean gravitational potential):

$\tau_{\mathrm{s}}=-\frac{e}{\dot{e}_{\mathrm{s}}} \times \frac{2 \pi}{\theta_{\mathrm{arm}}}$.

The factor $(2 \pi) /\left(\theta_{\mathrm{arm}}\right)$ (where $\theta_{\mathrm{arm}}$ is the angular width of the spiral arms at a given radius) accounts for the fact that, along an orbit, shocks dissipate energy only during the time spent by the gas in the spiral arm. Using ${ }^{13} \mathrm{CO}$ data, Turner \& Hurt (1992) fitted a rotation curve in the inner 320 pc of IC 342 of the form $v_{\varphi}(r)=a(\sqrt{1+b r}-1)$ (with $a, b$ constants); from the equation of motion, $v_{\varphi}^{2} / r=\mathrm{d} \phi(r) / \mathrm{d} r$, we can estimate $\phi(r)$ and $e$. The value of $e$ results to be $\simeq(2-3) \times v_{\varphi}^{2} / 2$. At the radius of GMC D $(r \sim 220 \mathrm{pc}) v_{\varphi} \sim 60 \mathrm{~km} \mathrm{~s}^{-1}$ and $\theta_{\text {arm }} /(2 \pi) \sim 0.1$, so we obtain $e \simeq 5 \times 10^{3} \mathrm{~km}^{2} \mathrm{~s}^{-2}$. The corresponding $\tau_{\mathrm{s}}$ is $\sim 16-160 \mathrm{Myr}$, i.e., $\sim 0.7-7$ rotations at the location of GMC D.

Our estimates suggest that the energy could be drained efficiently from the gas by large-scale shocks along the spiral arms. While it is true that the inflow of gas is mostly constrained by the angular momentum transfer, rather than by the energy dissipation rate (draining angular momentum is more difficult), large-scale shocks could have a non-negligible influence on the dissipation of energy of the gas on its way to the nucleus.

\section{References}

Athanassoula, E. 1992, MNRAS, 259, 345

Bachiller, R., Liechti, S., Walmsley, C. M., \& Colomer, F. 1995, A\&A, 295, L51

Bachiller, R., \& Pérez Gutiérrez, M. 1997, ApJ, 487, L93

Bachiller, R., Pérez Gutiérrez, M., Kumar, M. S. N., \& Tafalla, M. 2001, A\&A, 372, 899

Bauer, F. E., Brandt, W. N., \& Lehmer, B. 2003, AJ, 126, 2797

Becklin, E. E., Gatley, I., Matthews, K., et al. 1980, ApJ, 236, 441

Bergin, E. A., Melnick, G. J., \& Neufeld, D. A. 1998, ApJ, 499, 777

Böker, T., Forster-Schreiber, N. M., \& Genzel, R. 1997, AJ, 114, 1883

Böker, T., van der Marel, R. P., \& Vacca, W.D. 1999, AJ, 118, 831

Bonnell, A., Dobbs, C. L., Robitaille, T. P., \& Pringle, J. E. 2005, MNRAS, in press [arXiv: astro-ph/0509809]

Bregman, J. N., Cox, C. V., \& Tomisaka, K. 1993, ApJ, 415, L79

Buta, R. J., \& McCall, M. L. 1999, ApJS, 124, 33

Caselli, P., Hartquist, T. W., \& Havnes, O. 1997, A\&A, 322, 296

Casoli, F., \& Combes, F. 1982, A\&A, 110, 287

Cesaroni, R., Felli, M., Jenness, T., et al. 1999, A\&A, 345, 949

Codella, C., \& Bachiller, R. 1999, A\&A, 350, 659

Combes, F., \& Gerin, M. 1985, A\&A, 150, 327

Condon, J. J., Condon, M. A., Gisler, G., \& Puschell, J. J. 1982, ApJ, 252,102

Crosthwaite, L. P., Turner, J. L., Hurt, R. L., et al. 2001, AJ, 122, 797

Downes, D., Radford, S. J. E., Guilloteau, S., et al. 1992, A\&A, 262, 424

Draine, B. T., \& McKee, C. F. 1993, ARA\&A, 31, 373

Falgarone, E., \& Puget, J. L. 1985, A\&A, 142, 157

Fuente, A., García-Burillo, S., Gerin, M., et al. 2005a, ApJ, 619, L155

Fuente, A., Rizzo, J. R., Caselli, P., Bachiller, R., \& Henkel, C. 2005b, A\&A, 433, 535

Garay, G., Mardones, D., \& Rodríguez, L. F. 2000, ApJ, 545, 861

García-Burillo, S., Martín-Pintado, J., Fuente, A., \& Neri, R. 2000, A\&A, 355, 499

García-Burillo, S., \& Martín-Pintado, J. 2001a, The Promise of the Herschel Space Observatory, ESA SP-460, 163
García-Burillo, S., Martín-Pintado, J., Fuente, A., \& Neri, R. 2001b, ApJ, 563, L27

García-Burillo, S., Martín-Pintado, J., Fuente, A., Usero, A., \& Neri, R. 2002, ApJ, 575, L55

García-Burillo, S., Combes, F., Schinnerer, E., Boone, F., \& Hunt, L. K. 2005, A\&A, 441, 1011

Genzel, R., Lutz, D., Sturm, E., et al. 1998, ApJ, 498, 579

Gueth, F., Guilloteau, S., Dutrey, A., \& Bachiller, R. 1997, A\&A, 323, 943

Haas, M., Chini, R., \& Klaas, U. 2005, A\&A, 433, L17

Henkel, C., Chin, Y.-N., Mauersberger, R., \& Whiteoak, J. B. 1998, A\&A, 329, 443

Ho, P. T. P., Martin, R. N., Turner, J. L., \& Jackson, J. M. 1990, ApJ, 355, L19

Hüttemeister, S., Dahmen, G., Mauersberger, R., et al. 1998, A\&A, 334, 646

Ishizuki, S., Kawabe, R., Ishiguro, M., Okumura, S. K., \& Morita, K. 1990, Nature, 344, 224

Israel, F. P., \& Baas, F. 2003, A\&A, 404, 495

Jog, C. J., \& Solomon, P. M. 1992, ApJ, 387, 152

Jørgensen, J. K., Hogerheijde, M. R., Blake, G. A., et al. 2004, A\&A, 415,1021

Kennicutt, R. C. 1998, ApJ, 498, 541

Kimura, T., \& Tosa, M. 1996, A\&A, 308, 979

Levine, D. A., Turner, J. L., \& Hurt, R. L. 1994, Astronomy with Millimeter and Submillimeter Wave Interferometry, IAU Coll., 140, ASP Conf. Ser., 59, 339

Lo, K. Y., Berge, G. L., Claussen, M. J., et al. 1984, ApJ, 282, L59

Martín, S., Mauersberger, R., Martín-Pintado, J., García-Burillo, S., \& Henkel, C. 2003, A\&A, 411, L465

Martín, S., Martín-Pintado, J., Mauersberger, R., Henkel, C., \& García-Burillo, S. 2005, ApJ, 620, 210

Martín-Pintado, J., Bachiller, R., \& Fuente, A. 1992, A\&A, 254, 315

Martín-Pintado, J., de Vicente, P., Fuente, A., \& Planesas, P. 1997, ApJ, 482, L45

Martín-Pintado, J., Rizzo, J. R., de Vicente, P., Rodríguez-Fernández, N. J., \& Fuente, A. 2001, ApJ, 548, L65

Mauersberger, R., \& Henkel, C. 1991, A\&A, 245, 457

Mauersberger, R., \& Henkel, C. 1993, Rev. Mod. Astron., 6, 69

Meier, D. S., \& Turner, J. L. 2001, ApJ, 551, 687

Meier, D. S., \& Turner, J. L. 2005, ApJ, 618, 259

Mitchell, G. F., Johnstone, D., Moriarty-Schieven, G., Fich, M., \& Tothill, N. F. H. 2001, ApJ, 556, 215

Nguyen-Q-Rieu, Jackson, J. M., Henkel, C., Truong, B., \& Mauersberger, R. 1992, ApJ, 399, 521

Ogle, P. M., Brookings, T., Canizares, C. R., Lee, J. C., \& Marshall, H. L. 2003, A\&A, 402, 849

Reipurth, B., Rodríguez, L. F., \& Chini, R. 1999, AJ, 118, 983

Rieke, G. H., Cutri, R. M., Black, J. H., et al. 1985, ApJ, 290, 116

Rieke, G. H., Lebofsky, M. J., \& Walker, C. E. 1988, ApJ, 325, 679

Roberts, W. W. 1969, ApJ, 158, 123

Sage, L. J., \& Ziurys, L. M. 1995, ApJ, 447, 625

Saha, A., Claver, J., \& Hoessel, J. G. 2002, AJ, 124, 839

Schilke, P., Walmsley, C. M., Pineau des Forets, G., \& Flower, D. R. 1997, A\&A, 321, 293

Schinnerer, E., Böker, T., \& Meier, D. S. 2003, ApJ, 591, L115

Schulz, A., Güsten, R., Köster, B., \& Krause, D. 2001, A\&A, 371, 25

Tacconi, L. J., Genzel, R., Blietz, M., et al. 1994, ApJ, 426, L77

Turner, J. L., \& Ho, P. T. P. 1983, ApJ, 268, L79

Turner, J. L., \& Hurt, R. L. 1992, ApJ, 384, 72

Usero, A., García-Burillo, S., Fuente, A., Martín-Pintado, J., \& Rodríguez-Fernández, N. J. 2004, A\&A, 419, 897 